\title{
Reaction mechanisms of anisole pyrolysis at different temperatures: Experimental and theoretical studies
}

\section{Supporting Information}

Ting Zhang ${ }^{1,2}$, Chiranjivi Bhattarai ${ }^{2}$, Yeongkwon $\operatorname{Son}^{2}$, Vera Samburova ${ }^{2}$, Andrey Khlystov²*, Sergey A. Varganov ${ }^{1 *}$

${ }^{1}$ Department of Chemistry, University of Nevada, Reno, 1664 N. Virginia Street, Reno, Nevada 89557-0216, United States

${ }^{2}$ Organic Analytical Laboratory, Division of Atmospheric Sciences, Desert Research Institute, 2215 Raggio Parkway, Reno, 89512 Nevada, United States

${ }^{*}$ Corresponding Authors:

svarganov@unr.edu

andrey.khlystov@dri.edu 


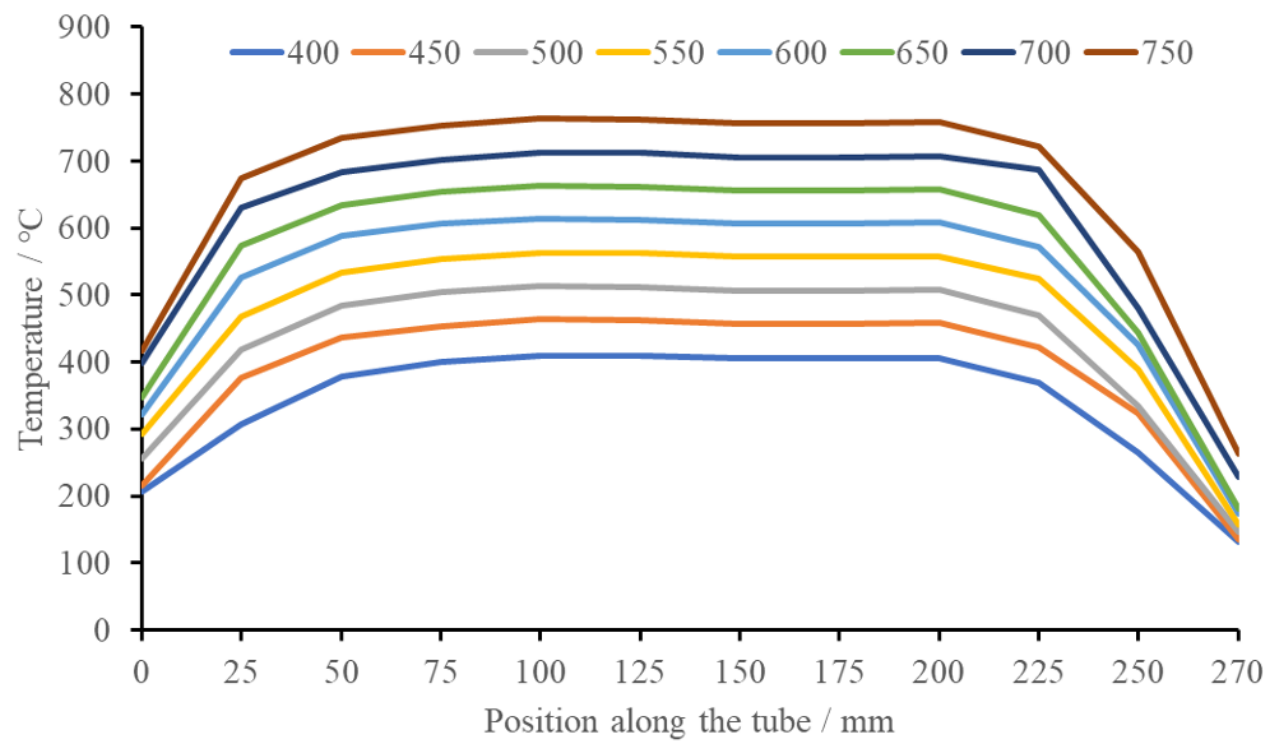

Figure S1. Temperature profile along the axis of the quartz tube. Different colors correspond to different target temperatures. 


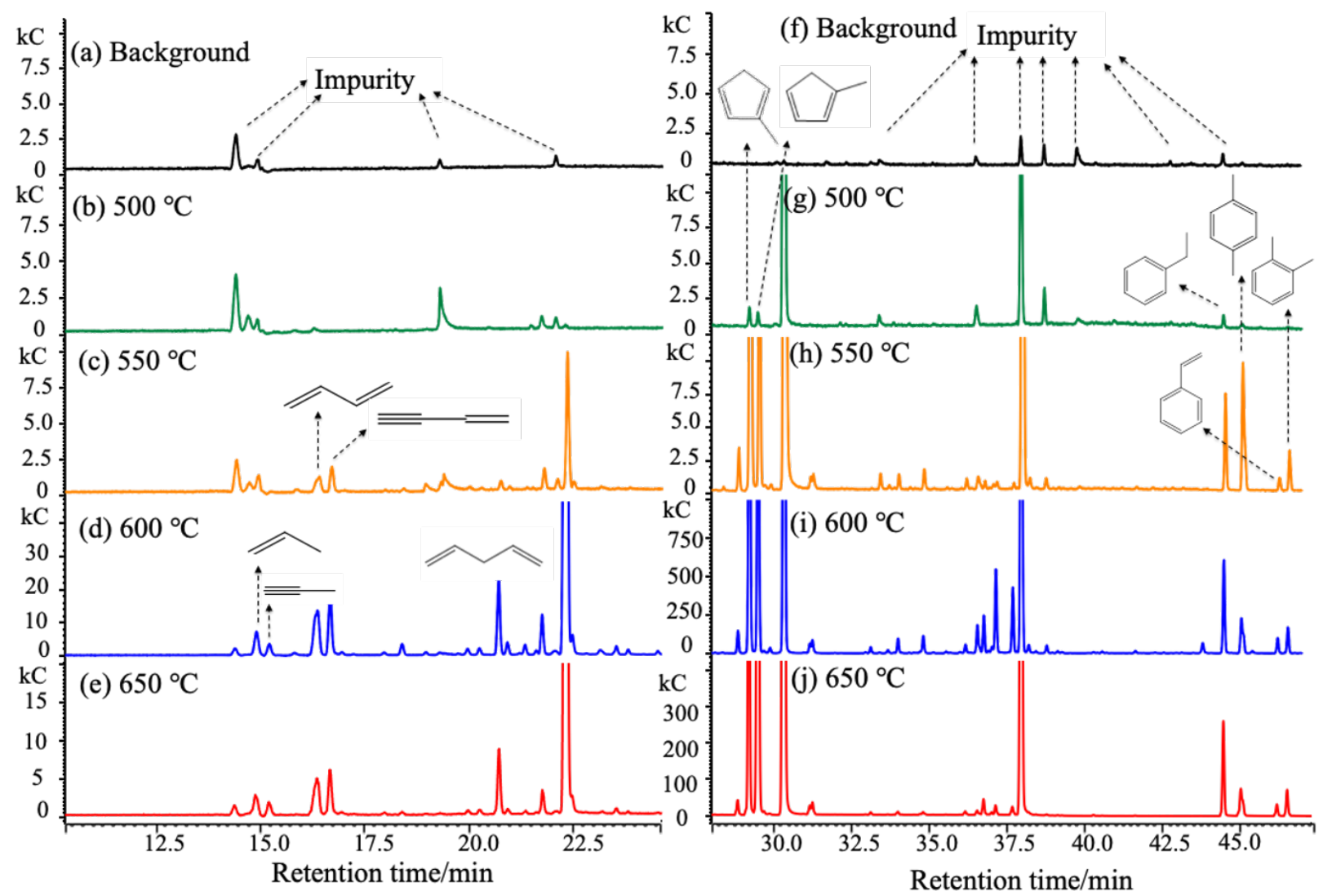

Figure S2. Part of GC-MS chromatograms of anisole decomposition products at $500-650{ }^{\circ} \mathrm{C}$. Vertical axis shows the total ion count. Chromatograms of the same color show the same samples with different retention time: from about 10 to $25 \mathrm{~min}$ for (a-e), and from about 27 to $47 \mathrm{~min}$ for $(\mathrm{f}-\mathrm{j})$. Traces of impurity in anisole are marked in (a) and (f). 

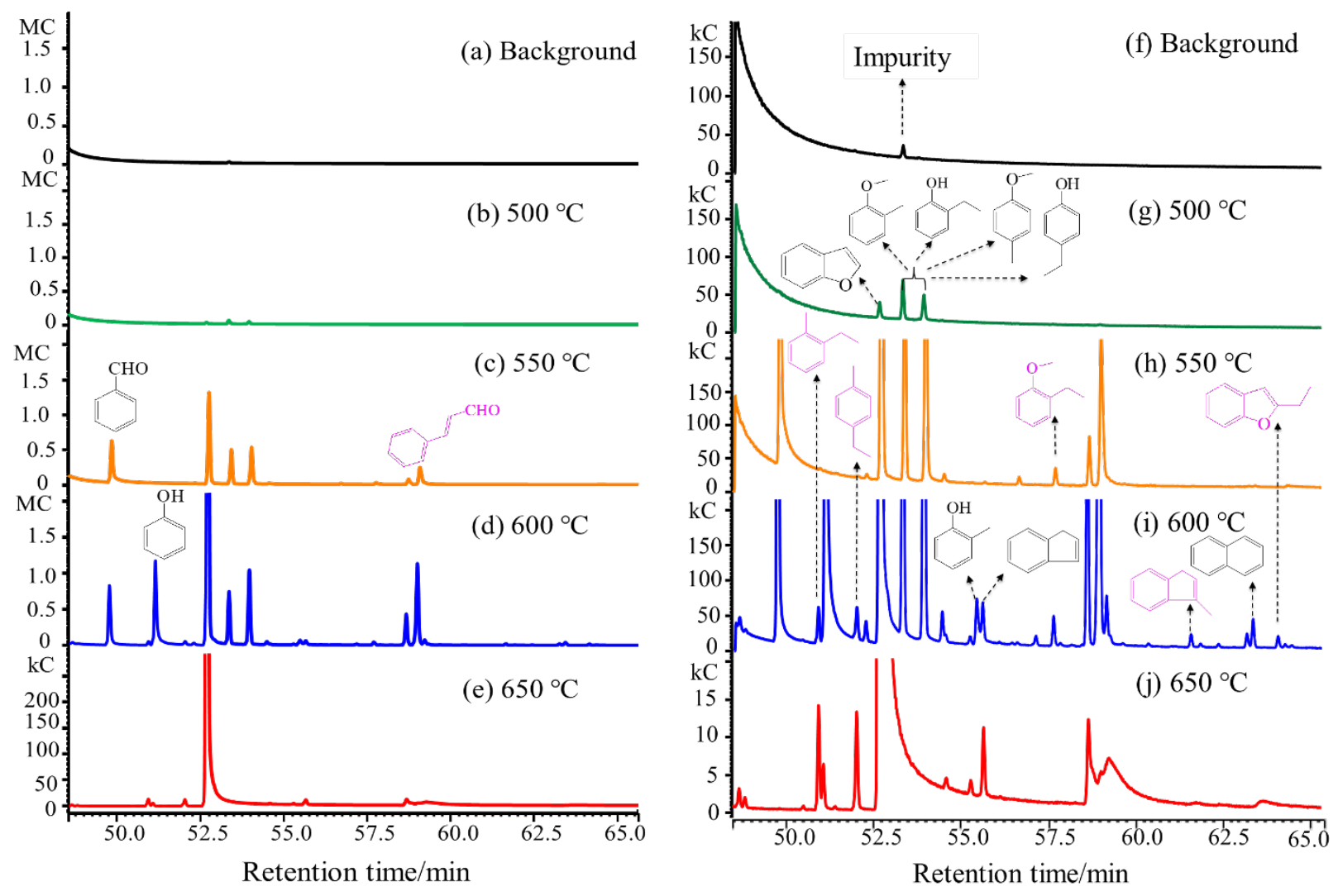

Figure S3. Part of GC-MS chromatograms of anisole decomposition products at $500-650{ }^{\circ} \mathrm{C}$. Vertical axis shows the total ion count. Left and right panels show the same chromatograms with different vertical scale. Traces of impurity in anisole are marked in (f). Compounds marked pink are assigned based on the NIST mass spectral database. ${ }^{(1)}$ 


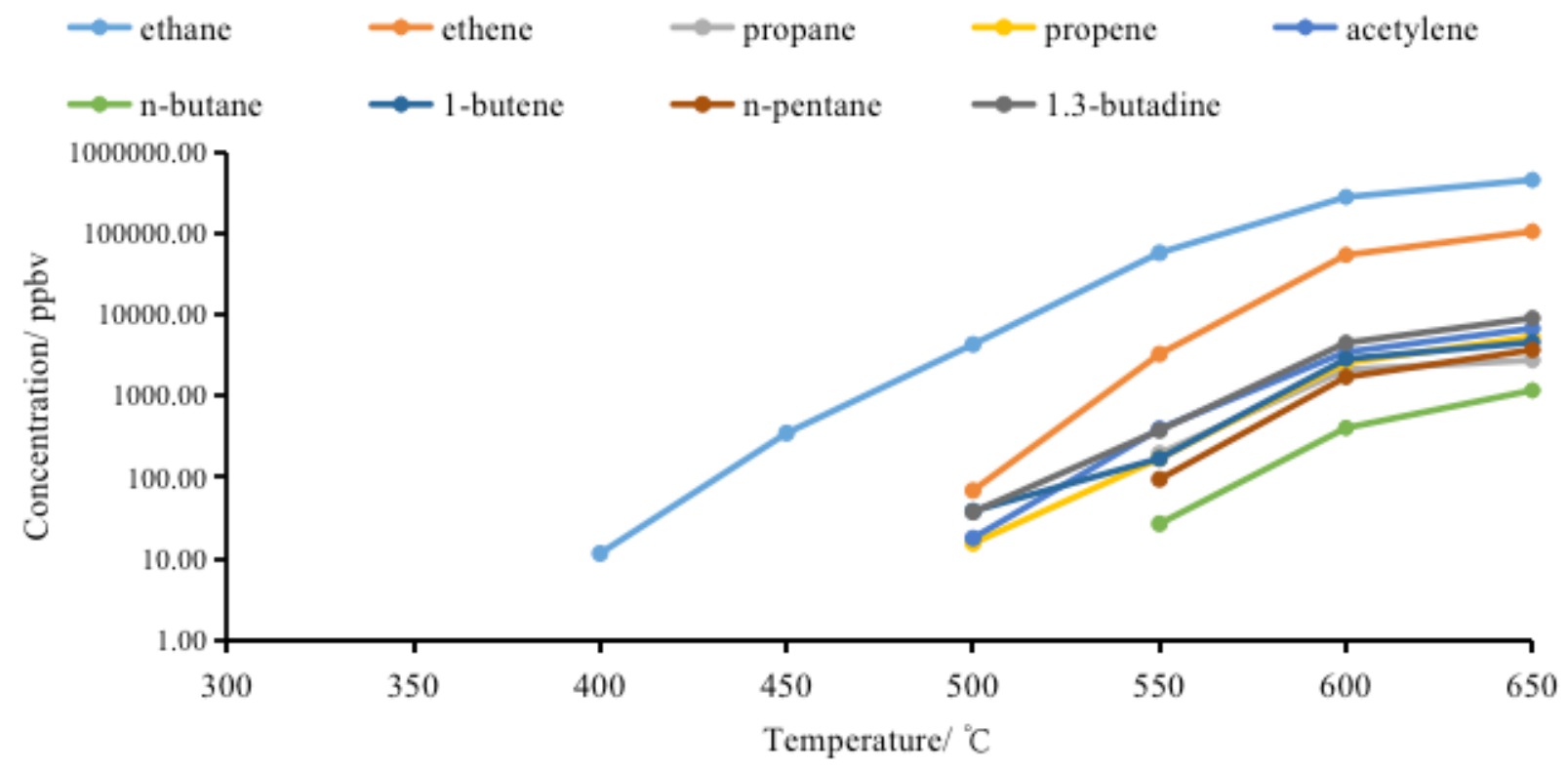

Figure S4. Concentrations of C2-C5 volatile organic compounds detected by FID as functions of temperature. 

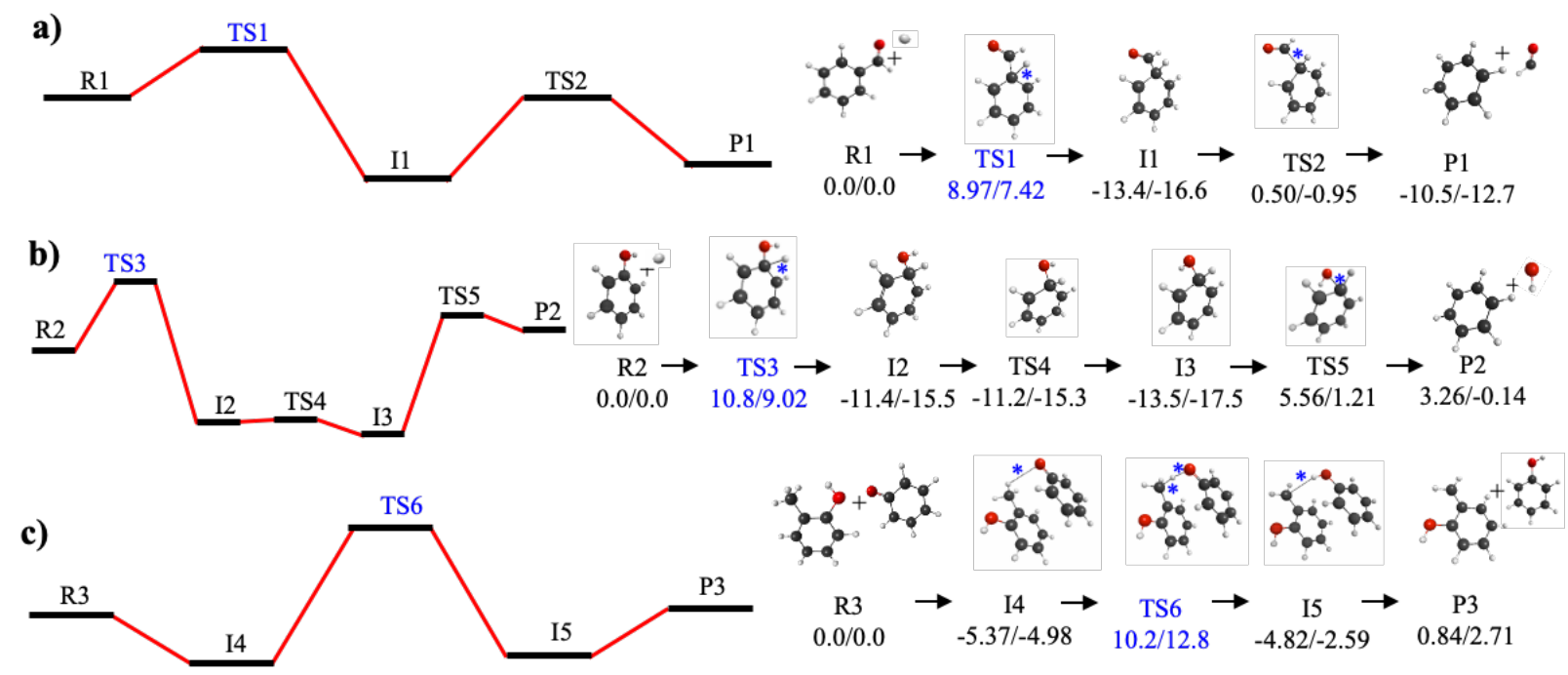

Figure S5. Reaction pathways for formation of benzene ( $a$ and b) and phenol (c): a) benzaldehyde $+\mathrm{H}, \mathrm{b}$ ) phenol $+\mathrm{H}$, and c) o-cresol + phenoxy. The energies $(\mathrm{kcal} / \mathrm{mol})$ are reported in the format $\mathrm{E}_{\mathrm{M} 06-2 \mathrm{X}} / \mathrm{E}_{\mathrm{F} 12-}$ $\operatorname{CCSDT}(\mathrm{T})$. The energy of the highest TS for each reaction pathway is marked blue. Asterisks $(*)$ indicate elongated bonds. 

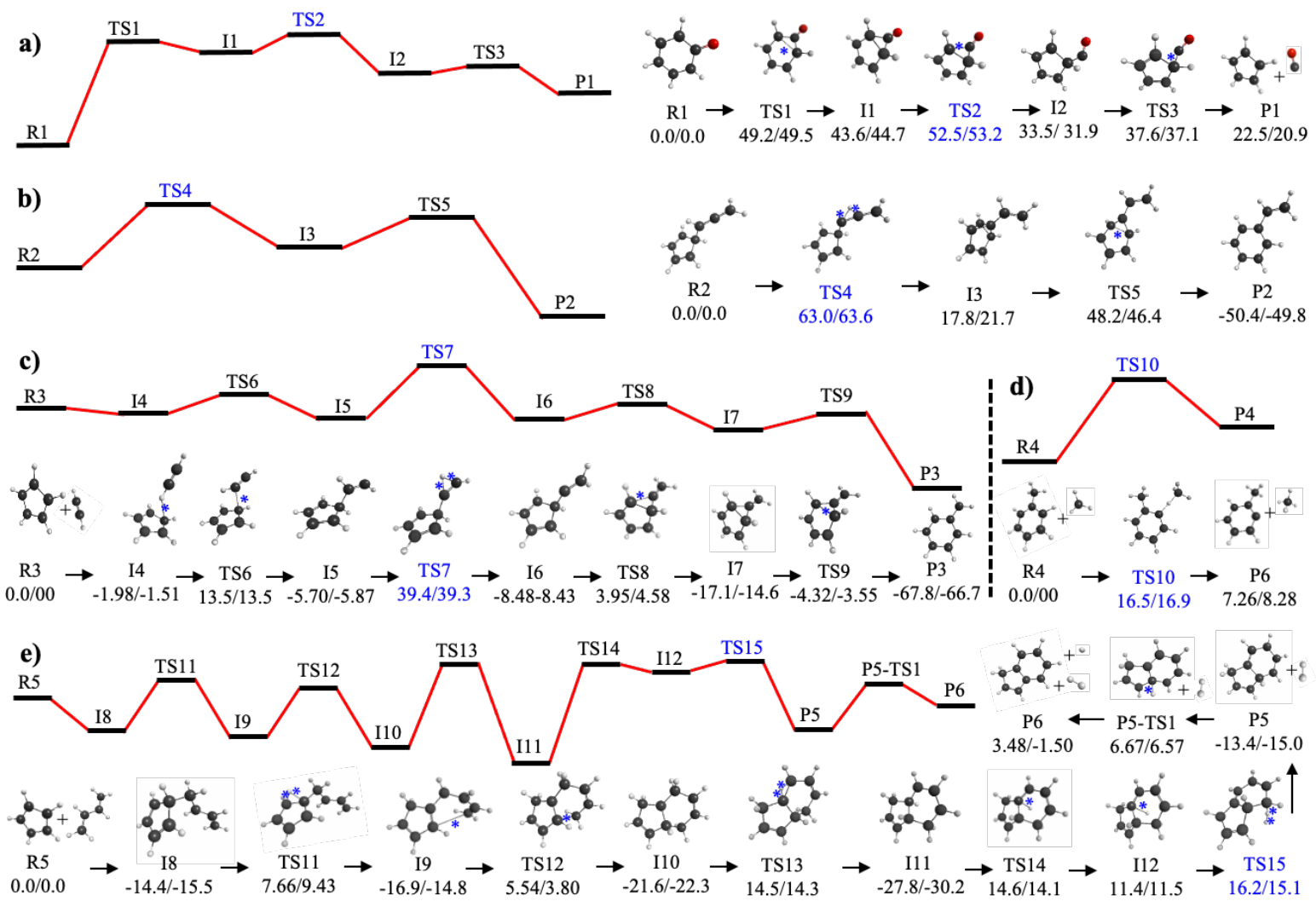

Figure S6. Reaction pathways for the dissociation of phenoxy to form cyclopentadienyl $+\mathrm{CO}$ (a), isomerization of 5-propadienylcyclopentadiene to form styrene (b), cyclopentadiene $+\mathrm{C}_{2} \mathrm{H}_{2}$ to form benzyl (c), toluene $+\mathrm{CH}_{3}$ to form o-methylphenyl $+\mathrm{CH}_{4}$ (d), and cyclopentadienyl $+\mathrm{C}_{4} \mathrm{H}_{6}$ to form indene $+\mathrm{H}_{2}+\mathrm{H}$

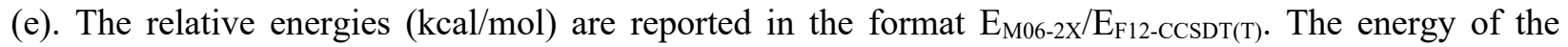
highest TS for each reaction pathway is marked blue. Asterisks $(*)$ indicate elongated bonds. 

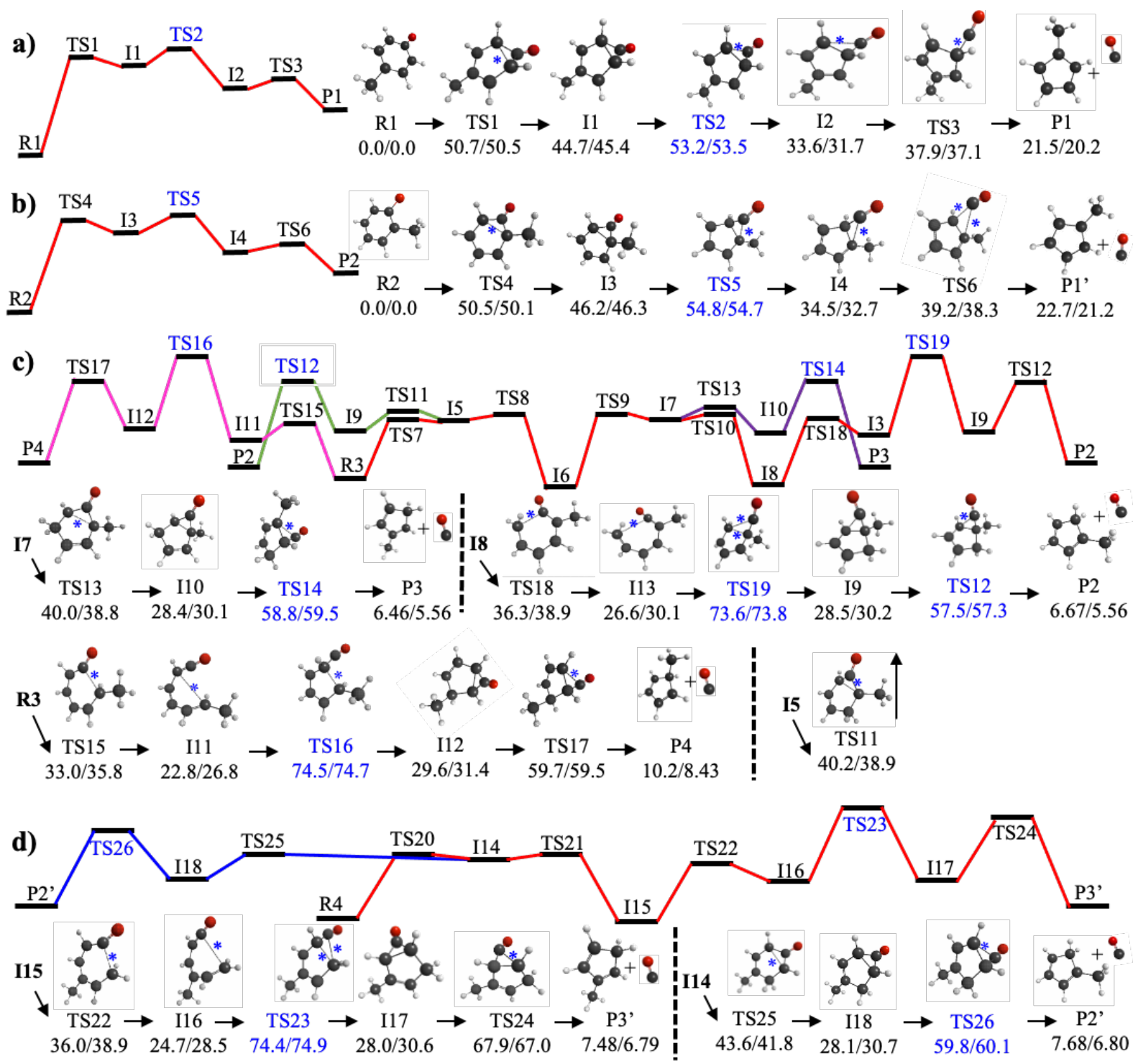

Figure S7. Reaction pathways for formation of methylcyclopentadienyl (a-b) and three isomeric methylcyclopentadiene (c-d): a) dissociation of p-methylphenoxy, b) dissociation of o-methylphenoxy c) dissociation of o-methylcyclohexadienone, and d) dissociation of p-methylcyclohexadienone. The relative energies $(\mathrm{kcal} / \mathrm{mol})$ are reported in the format $\mathrm{E}_{\mathrm{M} 06-2 \mathrm{X}} / \mathrm{E}_{\mathrm{F} 12-\mathrm{CCSDT}(\mathrm{T})}$. The energy of the highest TS for each reaction pathway is marked blue. Asterisks (*) indicate elongated bonds. The structures and energies of stationary points ( $\mathrm{R} 3 \rightarrow \mathrm{TS} 7 \rightarrow \mathrm{I} 5 \rightarrow \mathrm{TS} 8 \rightarrow \mathrm{I} 6 \rightarrow \mathrm{TS} 9 \rightarrow \mathrm{I} 7 \rightarrow \mathrm{TS} 10 \rightarrow \mathrm{I} 8 \quad$ in $\quad$ (c) and $(\mathrm{R} 4 \rightarrow \mathrm{TS} 20 \rightarrow \mathrm{I} 14 \rightarrow \mathrm{TS} 21 \rightarrow \mathrm{I} 15) \quad$ in $\quad(\mathrm{d})$ are the same as points $(\mathrm{R} 1 \rightarrow \mathrm{TS} 1 \rightarrow \mathrm{I} 1 \rightarrow \mathrm{TS} 2 \rightarrow \mathrm{I} 2 \rightarrow \mathrm{TS} 3 \rightarrow \mathrm{I} 3 \rightarrow \mathrm{TS} 4 \rightarrow \mathrm{I} 4)$ in Figure $9 \mathrm{a}$ and $(\mathrm{R} 2 \rightarrow \mathrm{TS} 7 \rightarrow \mathrm{I} 6 \rightarrow \mathrm{TS} 8 \rightarrow \mathrm{I} 7)$ in Figure $9 \mathrm{~b}$, respectively. 
Table S1. Appearance of each compound at different temperatures and the confirmation confidence by standard reference or NIST probability in GC-MS.

\begin{tabular}{|c|c|c|c|c|c|c|c|c|c|c|}
\hline${ }_{\text {Compound }}^{\text {Temperature/ }}{ }^{\circ} \mathrm{C}$ & 300 & 350 & 400 & 450 & 500 & 550 & 600 & 650 & $\begin{array}{l}\text { Std. } \\
\text { ref. }\end{array}$ & $\begin{array}{l}\text { NIST } \\
\text { prob. } \\
(\%)\end{array}$ \\
\hline anisole & + & + & + & + & + & + & + & + & + & \\
\hline benzene & - & - & + & + & + & + & + & + & + & \\
\hline toluene & - & - & - & + & + & + & + & + & + & \\
\hline benzofuran & - & - & - & - & + & + & + & + & & 33.2 \\
\hline o/p-methylanisole & - & - & - & - & + & + & + & - & & $46.6 / 35.5$ \\
\hline 1-methylcyclopentadiene & - & - & - & - & + & + & + & + & & 34.3 \\
\hline 2-methylcyclopentadiene & - & - & - & - & + & + & + & + & & 18.5 \\
\hline benzaldehyde & - & - & - & - & - & + & + & - & & 37.9 \\
\hline o-ethylanisole & - & - & - & - & - & + & + & - & & 47.1 \\
\hline cinnamaldehyde & - & - & - & - & - & + & + & + & & 35.2 \\
\hline 1,3-cyclopentadiene & - & - & - & - & - & + & + & + & & 42.1 \\
\hline ethylbenzene & - & - & - & - & - & + & + & + & + & \\
\hline styrene & - & - & - & - & - & + & + & + & + & \\
\hline o-xylene & - & - & - & - & - & + & + & + & + & \\
\hline p-xylene & - & - & - & - & - & + & + & + & + & \\
\hline phenol & - & - & - & - & - & - & + & - & & \\
\hline $\mathrm{o} / \mathrm{p}$-cresol & - & - & - & - & - & - & + & - & & $31.7 / 18.3$ \\
\hline indene & - & - & - & - & - & - & + & + & & 3.58 \\
\hline naphthalene & - & - & - & - & - & - & + & + & & 45.9 \\
\hline 1-ethyl-2-methylbenzene & - & - & - & - & - & - & + & + & & 24.9 \\
\hline 1-ethyl-4-methylbenzene & - & - & - & - & - & - & + & + & & 25.5 \\
\hline 3-methyl-1H-indene & - & - & - & - & - & - & + & - & & 11.2 \\
\hline ethyl-2-benzofuran & - & - & - & - & - & - & + & - & & 69.2 \\
\hline propene $\mathrm{C}_{3} \mathrm{H}_{6}$ & - & - & - & - & - & - & + & + & + & 38.3 \\
\hline propyne $\mathrm{C}_{3} \mathrm{H}_{4}$ & - & - & - & - & - & - & + & + & & 46.6 \\
\hline 1,3-butadiene $\mathrm{C}_{4} \mathrm{H}_{6}$ & - & - & - & - & - & + & + & + & + & 12.5 \\
\hline vinylacetylene $\mathrm{C}_{4} \mathrm{H}_{4}$, & - & - & - & - & - & + & + & + & & 97.9 \\
\hline 1,4-pentadiene $\mathrm{C}_{5} \mathrm{H}_{8}$ & - & - & - & - & - & + & + & + & & 20.8 \\
\hline
\end{tabular}


Table S2. Cartesian coordinates $(\AA)$ of the reactants, highest energy TSs and products for pathways of the formation of benzene and benzaldehyde shown in Figure 6.

\begin{tabular}{|c|c|c|c|c|c|c|c|}
\hline \multicolumn{4}{|c|}{$\mathrm{R} 1$} & \multicolumn{4}{|c|}{ TS1 } \\
\hline & & Anisole & & $\mathrm{C}$ & -1.07201886 & 0.53498626 & 0.65002614 \\
\hline $\mathrm{C}$ & -1.03408098 & 0.56278247 & 0.58589780 & $\mathrm{C}$ & 0.08497704 & -0.25047088 & 0.42726436 \\
\hline $\mathrm{C}$ & 0.07928642 & -0.26169351 & 0.46324497 & $\mathrm{C}$ & -0.07563878 & -1.59427750 & 0.01269660 \\
\hline $\mathrm{C}$ & -0.07121084 & -1.56868327 & -0.00086180 & $\mathrm{C}$ & -1.31672609 & -2.05356193 & -0.36079285 \\
\hline $\mathrm{C}$ & -1.32421863 & -2.04241705 & -0.33710435 & $\mathrm{C}$ & -2.43913913 & -1.22963750 & -0.26558262 \\
\hline $\mathrm{C}$ & -2.44543147 & -1.22559977 & -0.21772397 & $\mathrm{C}$ & -2.30573726 & 0.04989411 & 0.26039112 \\
\hline $\mathrm{C}$ & -2.28979087 & 0.06969099 & 0.24266809 & $\mathrm{H}$ & -0.98138183 & 1.53390038 & 1.05111814 \\
\hline $\mathrm{H}$ & -0.94004178 & 1.57796109 & 0.94286722 & $\mathrm{O}$ & 1.30510855 & 0.05228843 & 0.95491815 \\
\hline $\mathrm{O}$ & 1.34650898 & 0.11553852 & 0.76956660 & $\mathrm{H}$ & 0.80860722 & -2.21418118 & -0.04556207 \\
\hline $\mathrm{H}$ & 0.81145781 & -2.18837738 & -0.08690408 & $\mathrm{H}$ & -1.42178321 & -3.06860209 & -0.72157842 \\
\hline $\mathrm{H}$ & -1.42962313 & -3.05827546 & -0.69591814 & $\mathrm{H}$ & -3.41108990 & -1.59660292 & -0.56514537 \\
\hline $\mathrm{H}$ & -3.42549634 & -1.59900045 & -0.48157707 & $\mathrm{H}$ & -3.17991948 & 0.67709619 & 0.37929326 \\
\hline $\mathrm{H}$ & -3.15202880 & 0.71692640 & 0.34065825 & $\mathrm{C}$ & 1.57140017 & 1.41445279 & 1.19818771 \\
\hline $\mathrm{C}$ & 1.54430723 & 1.42719948 & 1.24362361 & $\mathrm{H}$ & 1.34761274 & 2.01306248 & 0.30852643 \\
\hline $\mathrm{H}$ & 1.23367131 & 2.16808200 & 0.50169659 & $\mathrm{H}$ & 2.63107777 & 1.48539352 & 1.42863345 \\
\hline $\mathrm{H}$ & 2.61051202 & 1.52684724 & 1.42829132 & $\mathrm{H}$ & 0.99385244 & 1.79002845 & 2.04675245 \\
\hline $\mathrm{H}$ & 0.99707490 & 1.59853864 & 2.17471719 & $\mathrm{H}$ & 0.37235290 & 0.39311400 & -1.10069680 \\
\hline \multicolumn{8}{|c|}{$\mathrm{H}$} \\
\hline \multicolumn{4}{|c|}{$\mathrm{P} 1$} & \multicolumn{4}{|c|}{$\mathrm{R} 2$} \\
\hline \multicolumn{4}{|c|}{ Benzene } & \\
\hline $\mathrm{C}$ & -0.56262773 & 1.04913163 & 0.00000000 & \multicolumn{4}{|c|}{ Same with R1(Anisole) } \\
\hline $\mathrm{C}$ & 0.63989681 & 0.35511100 & -0.00000000 & \multicolumn{4}{|c|}{$\mathrm{CH}_{3}$} \\
\hline $\mathrm{C}$ & 0.63995177 & -1.03336751 & 0.00000000 & $\mathrm{C}$ & -0.42107907 & -0.19938767 & 0.13437793 \\
\hline $\mathrm{C}$ & -0.56263220 & -1.72752857 & 0.00000000 & $\mathrm{H}$ & -0.73210812 & 0.81623310 & -0.04520507 \\
\hline $\mathrm{C}$ & -1.76519418 & -1.03347826 & 0.00000000 & $\mathrm{H}$ & -0.73210508 & -0.70724636 & 1.03204978 \\
\hline $\mathrm{C}$ & -1.76512480 & 0.35504588 & 0.00000000 & $\mathrm{H}$ & 0.20081651 & -0.70724046 & -0.58382148 \\
\hline $\mathrm{H}$ & -0.56266338 & 2.13158131 & 0.00000000 & & & & \\
\hline $\mathrm{H}$ & 1.57737124 & 0.89616209 & -0.00000000 & & & & \\
\hline $\mathrm{H}$ & 1.57732844 & -1.57459891 & -0.00000000 & & & & \\
\hline $\mathrm{H}$ & -0.56243694 & -2.80995750 & 0.00000000 & & & & \\
\hline $\mathrm{H}$ & -2.70272803 & -1.57445562 & -0.00000000 & & & & \\
\hline $\mathrm{H}$ & -2.70251918 & $\begin{array}{l}0.89622587 \\
\mathrm{OCH}_{3}\end{array}$ & -0.00000000 & & & & \\
\hline $\mathrm{C}$ & -0.85211319 & 0.04802310 & 0.01826227 & & & & \\
\hline $\mathrm{O}$ & -0.78928572 & 1.41263664 & -0.02015566 & & & & \\
\hline $\mathrm{H}$ & 0.05500903 & -0.29900709 & -0.50528812 & & & & \\
\hline $\mathrm{H}$ & -0.80451876 & -0.34984502 & 1.03788054 & & & & \\
\hline $\mathrm{H}$ & -1.71131670 & -0.35272005 & -0.53069901 & & & & \\
\hline \multicolumn{4}{|c|}{ TS3 } & \multicolumn{4}{|c|}{ P2 } \\
\hline
\end{tabular}




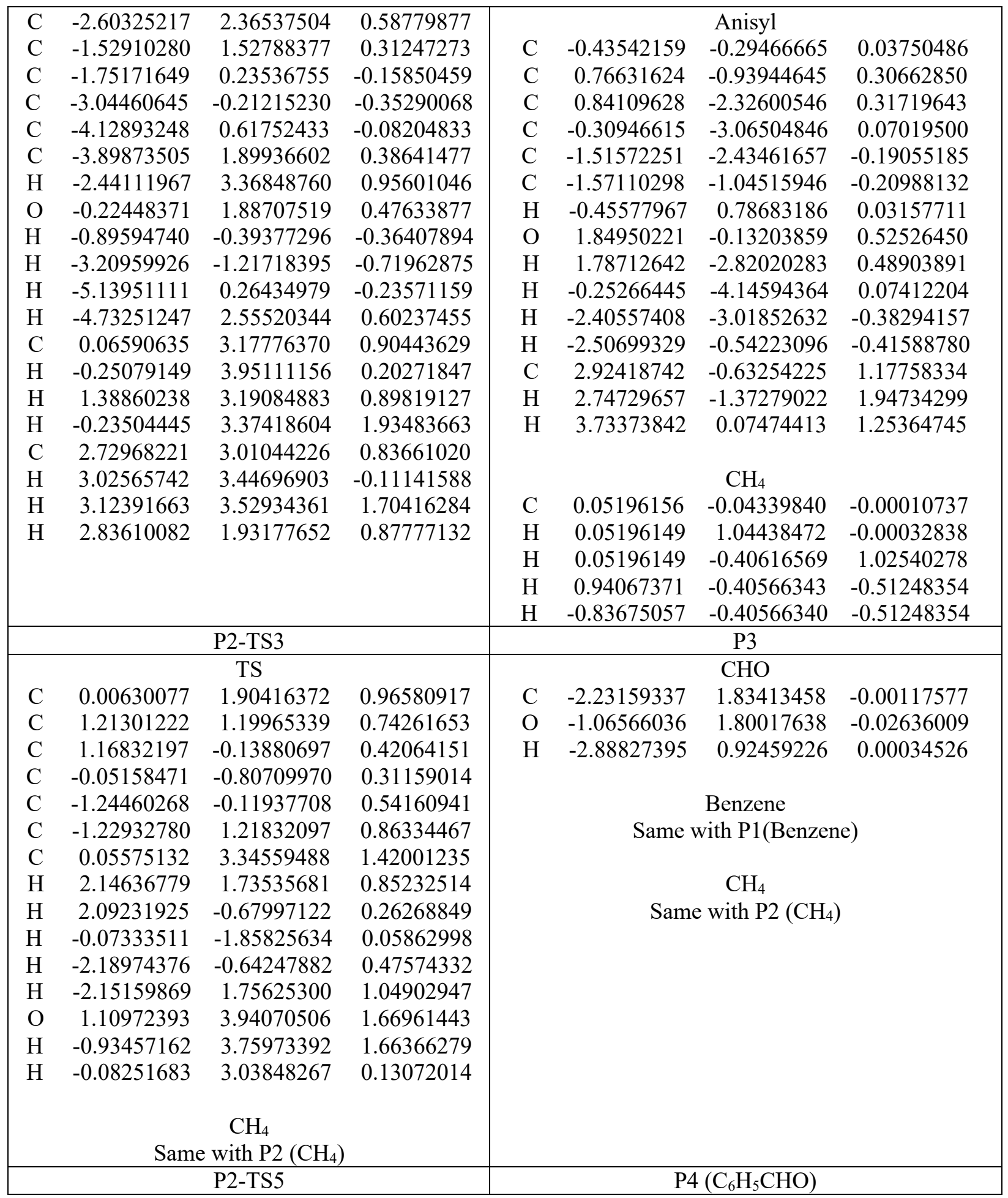




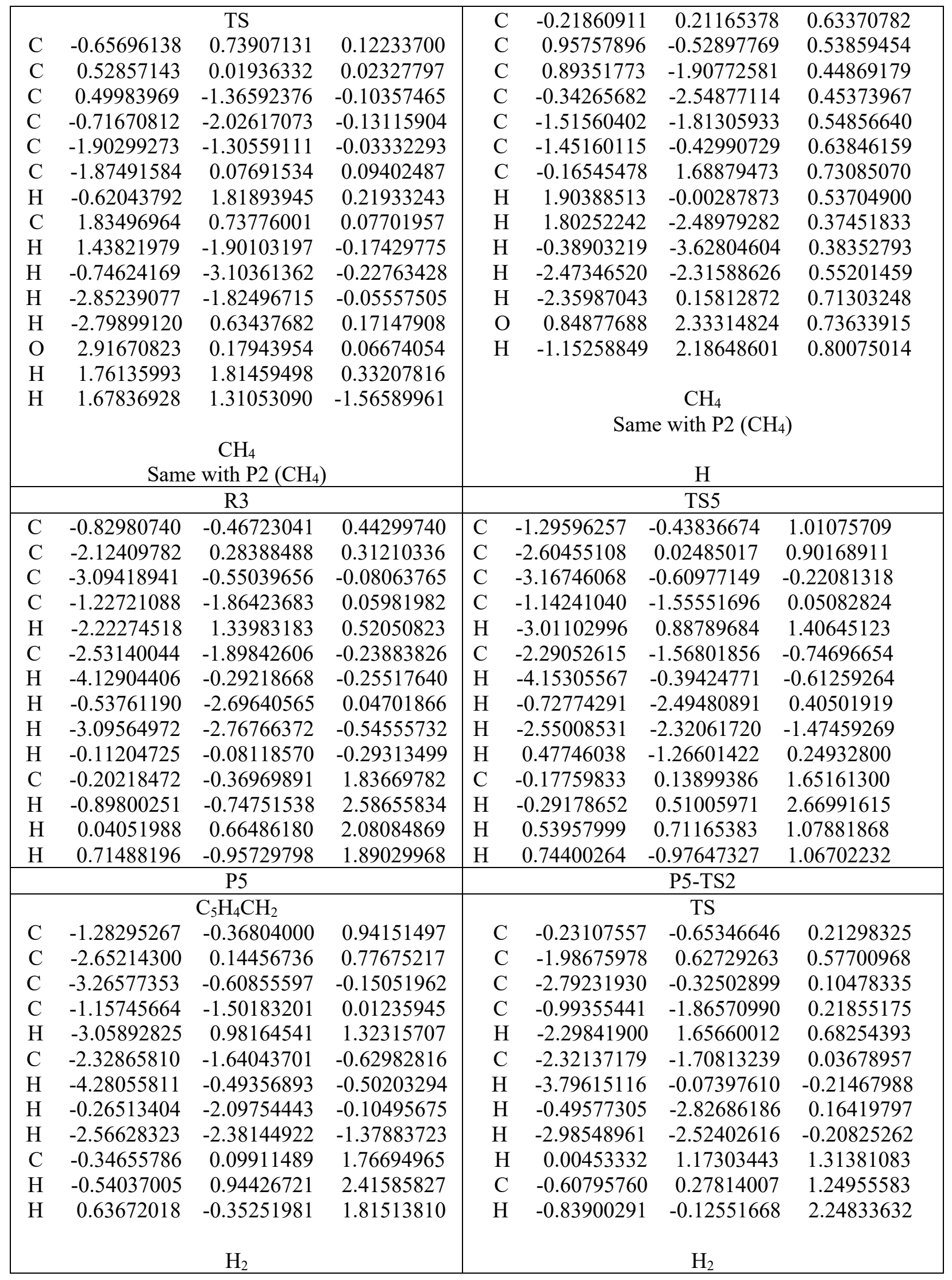




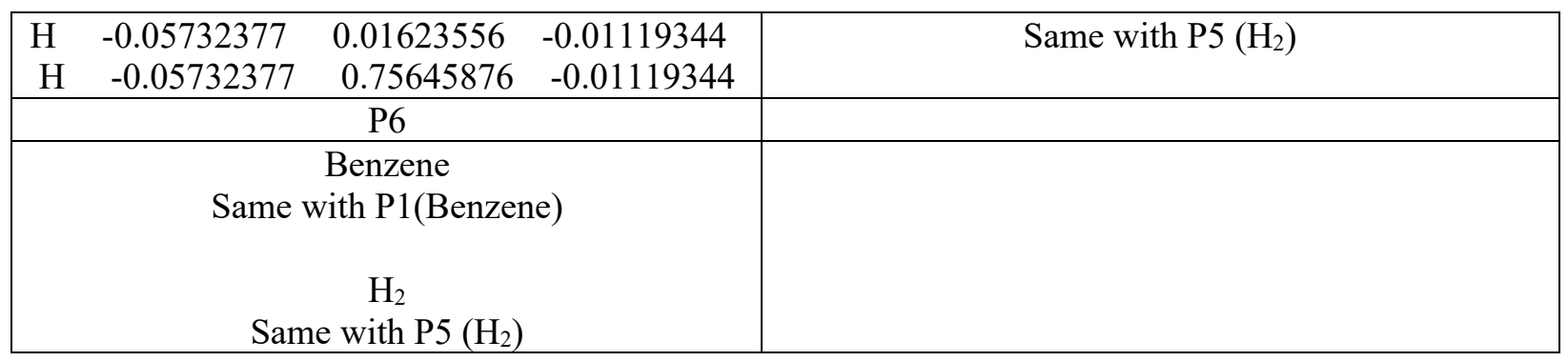


Table S3. Cartesian coordinates $(\AA)$ of the reactants, highest energy TSs and products for pathways of the formation of toluene and phenyl shown in Figure 7.

\begin{tabular}{|c|c|c|c|c|c|c|c|}
\hline \multicolumn{4}{|c|}{$\mathrm{R} 1$} & \multicolumn{4}{|c|}{ TS1 } \\
\hline & & Anisole & & $\mathrm{C}$ & -1.06553531 & 0.56936687 & 0.64926302 \\
\hline \multirow{2}{*}{\multicolumn{4}{|c|}{ Same with R1(Anisole) in Table S1 }} & $\mathrm{C}$ & 0.10276050 & -0.18440361 & 0.34135750 \\
\hline & & & & $\mathrm{C}$ & -0.06612689 & -1.55622077 & -0.00432924 \\
\hline \multicolumn{4}{|c|}{$\mathrm{CH}_{3}$} & $\mathrm{C}$ & -1.31292653 & -2.04277921 & -0.30538568 \\
\hline \multicolumn{4}{|c|}{ Same with $\mathrm{R} 2\left(\mathrm{CH}_{3}\right)$ in Table $\mathrm{S} 1$} & $\mathrm{C}$ & -2.44538689 & -1.23116565 & -0.17816839 \\
\hline & & & & $\mathrm{C}$ & -2.30636978 & 0.05763318 & 0.33004647 \\
\hline & & & & $\mathrm{H}$ & -0.97740734 & 1.57157850 & 1.04348505 \\
\hline & & & & $\mathrm{O}$ & 1.33491421 & 0.11055376 & 0.86743361 \\
\hline & & & & $\mathrm{H}$ & 0.82258826 & -2.16952801 & -0.07343692 \\
\hline & & & & $\mathrm{H}$ & -1.42064559 & -3.07158017 & -0.62454540 \\
\hline & & & & $\mathrm{H}$ & -3.42522454 & -1.61984527 & -0.41853264 \\
\hline & & & & $\mathrm{H}$ & -3.18564248 & 0.66710812 & 0.49638250 \\
\hline & & & & $\mathrm{C}$ & 1.53211224 & 1.41132176 & 1.36556745 \\
\hline & & & & $\mathrm{H}$ & 1.29480124 & 2.16951370 & 0.61338663 \\
\hline & & & & $\mathrm{H}$ & 2.58495188 & 1.48362362 & 1.62621307 \\
\hline & & & & $\mathrm{H}$ & 0.92688364 & 1.59053862 & 2.25856495 \\
\hline & & & & $\mathrm{C}$ & 0.40638012 & 0.54272395 & -1.60516918 \\
\hline & & & & $\mathrm{H}$ & 0.52483529 & 1.61295605 & -1.47981882 \\
\hline & & & & $\mathrm{H}$ & -0.50175822 & 0.23320478 & -2.10742640 \\
\hline & & & & $\mathrm{H}$ & 1.30795574 & 0.00473049 & -1.87147725 \\
\hline \multicolumn{4}{|c|}{$\mathrm{P} 1$} & \multicolumn{4}{|c|}{$\mathrm{R} 2$} \\
\hline \multicolumn{4}{|c|}{ Toluene } & \multicolumn{4}{|c|}{ o-cresol } \\
\hline $\mathrm{C}$ & -3.15687537 & 2.85536170 & 0.07636634 & $\mathrm{C}$ & -3.52482939 & 1.07518399 & -0.14104095 \\
\hline $\mathrm{C}$ & -2.37406754 & 2.08458066 & 0.93126804 & $\mathrm{C}$ & -2.51961899 & 0.15957552 & 0.17894825 \\
\hline $\mathrm{C}$ & -2.51079369 & 0.70390683 & 0.97460175 & $\mathrm{C}$ & -2.80025744 & -1.19308174 & 0.31497759 \\
\hline $\mathrm{C}$ & -3.43831515 & 0.06971630 & 0.16078919 & $\mathrm{C}$ & -4.09499454 & -1.64930320 & 0.13115461 \\
\hline $\mathrm{C}$ & -4.22768593 & 0.82732040 & -0.69394457 & $\mathrm{C}$ & -5.11049795 & -0.75850868 & -0.18803884 \\
\hline $\mathrm{C}$ & -4.08661032 & 2.20677161 & -0.73283416 & $\mathrm{C}$ & -4.81555748 & 0.59029645 & -0.32020333 \\
\hline $\mathrm{H}$ & -1.89373267 & 0.12316186 & 1.64839220 & $\mathrm{H}$ & -1.14809120 & 1.49939561 & 0.25290903 \\
\hline $\mathrm{H}$ & -3.54856801 & -1.00628591 & 0.19472589 & $\mathrm{O}$ & -1.22759461 & 0.54835844 & 0.37068152 \\
\hline $\mathrm{H}$ & -4.95775223 & 0.34285772 & -1.32981062 & $\mathrm{H}$ & -1.99105906 & -1.86653101 & 0.56402415 \\
\hline $\mathrm{H}$ & -4.70871067 & 2.79279375 & -1.39976728 & $\mathrm{H}$ & -4.30924416 & -2.70473695 & 0.23833418 \\
\hline $\mathrm{C}$ & -2.98681951 & 4.34812498 & 0.00665871 & $\mathrm{H}$ & -6.12306833 & -1.10988438 & -0.33265641 \\
\hline $\mathrm{H}$ & -2.56639886 & 4.74103498 & 0.93196934 & $\mathrm{H}$ & -5.60338163 & 1.29188776 & -0.56895494 \\
\hline $\mathrm{H}$ & -2.31144786 & 4.62203121 & -0.80710864 & $\mathrm{C}$ & -3.20102310 & 2.53619576 & -0.28276590 \\
\hline $\mathrm{H}$ & -1.64973533 & 2.57305026 & 1.57290804 & $\mathrm{H}$ & -2.79317236 & 2.95164299 & 0.64355028 \\
\hline \multirow[t]{3}{*}{$\mathrm{H}$} & -3.93968511 & 4.84499693 & -0.17589664 & $\mathrm{H}$ & -4.09534216 & 3.10560036 & -0.52784491 \\
\hline & & & & $\mathrm{H}$ & -2.47172379 & 2.71386719 & -1.07879078 \\
\hline & \multicolumn{3}{|c|}{$\begin{array}{c}\mathrm{OCH}_{3} \\
\text { Same with P1 }\left(\mathrm{OCH}_{3}\right) \text { in Table S1 }\end{array}$} & \multicolumn{4}{|c|}{$\mathrm{H}$} \\
\hline \multicolumn{4}{|c|}{ TS3 } & \multicolumn{4}{|c|}{$\mathrm{P} 2$} \\
\hline $\mathrm{C}$ & -1.90353632 & 1.62733245 & -0.22818427 & & & $\mathrm{OH}$ & \\
\hline $\mathrm{C}$ & -0.91554379 & 0.69300085 & 0.18314572 & $\mathrm{O}$ & -1.26077867 & -0.06726857 & -0.00609807 \\
\hline $\mathrm{C}$ & -1.22911346 & -0.67766124 & 0.24752332 & $\mathrm{H}$ & -1.14327919 & 0.88999206 & 0.12377609 \\
\hline $\mathrm{C}$ & -2.53770399 & -1.08694327 & 0.10402500 & & & & \\
\hline $\mathrm{C}$ & -3.53635740 & -0.15890841 & -0.17584810 & & & & \\
\hline
\end{tabular}




\begin{tabular}{|c|c|c|c|c|c|c|c|}
\hline $\mathrm{C}$ & -3.20524907 & 1.18124413 & -0.35506997 & \multirow{2}{*}{\multicolumn{4}{|c|}{$\begin{array}{c}\text { Toluene } \\
\text { Same with P1 (Toluene) }\end{array}$}} \\
\hline $\mathrm{H}$ & 0.54375440 & 1.93404377 & 0.34997642 & & & & \\
\hline $\mathrm{O}$ & 0.41809523 & 1.00556064 & 0.12785995 & & & & \\
\hline $\mathrm{H}$ & -0.42858022 & -1.37319326 & 0.45924097 & & & & \\
\hline $\mathrm{H}$ & -2.78382111 & -2.13632846 & 0.19972546 & & & & \\
\hline $\mathrm{H}$ & -4.56320190 & -0.48032543 & -0.28491366 & & & & \\
\hline $\mathrm{H}$ & -3.97821808 & 1.89574611 & -0.61223149 & & & & \\
\hline $\mathrm{C}$ & -1.51731479 & 3.06211710 & -0.39725536 & & & & \\
\hline $\mathrm{H}$ & -1.12822294 & 3.46890330 & 0.54450452 & & & & \\
\hline $\mathrm{H}$ & -2.37409544 & 3.66610670 & -0.68873358 & & & & \\
\hline $\mathrm{H}$ & -0.74103868 & 3.18109107 & -1.15713966 & & & & \\
\hline $\mathrm{H}$ & -1.16512287 & 1.07228231 & 1.81743944 & & & & \\
\hline \multicolumn{4}{|c|}{ R3 } & \multicolumn{4}{|c|}{ TS6 } \\
\hline & & p-cresol & & $\mathrm{C}$ & -1.89962900 & 1.61274159 & -0.23455498 \\
\hline $\mathrm{C}$ & -0.77442867 & 0.56739199 & 1.14350688 & $\mathrm{C}$ & -0.90913433 & 0.70726961 & 0.20385782 \\
\hline $\mathrm{C}$ & 0.19254637 & -0.41231620 & 0.97621721 & $\mathrm{C}$ & -1.23169196 & -0.66626501 & 0.25796026 \\
\hline $\mathrm{C}$ & -0.03042277 & -1.45567429 & 0.08438993 & $\mathrm{C}$ & -2.53987527 & -1.06406283 & 0.10902592 \\
\hline $\mathrm{C}$ & -1.21568370 & -1.50680757 & -0.62627137 & $\mathrm{C}$ & -3.55990195 & -0.14824378 & -0.17216726 \\
\hline $\mathrm{C}$ & -2.20404172 & -0.53422481 & -0.47341323 & $\mathrm{C}$ & -3.20511508 & 1.18659496 & -0.36630711 \\
\hline $\mathrm{C}$ & -1.95918894 & 0.49935302 & 0.42076704 & $\mathrm{H}$ & 0.53467745 & 1.97040403 & 0.24355933 \\
\hline $\mathrm{H}$ & -0.60362470 & 1.38472593 & 1.83561552 & $\mathrm{O}$ & 0.42391559 & 1.01834035 & 0.15955456 \\
\hline $\mathrm{O}$ & 1.37573624 & -0.40385729 & 1.65234566 & $\mathrm{H}$ & -0.43923292 & -1.37059116 & 0.47114918 \\
\hline $\mathrm{H}$ & 0.73452127 & -2.21081781 & -0.03797500 & $\mathrm{H}$ & -2.78619909 & -2.11570024 & 0.20067643 \\
\hline $\mathrm{H}$ & -1.37938201 & -2.32331848 & -1.32059133 & $\mathrm{H}$ & -3.96933532 & 1.90276730 & -0.64424306 \\
\hline $\mathrm{H}$ & 1.41044068 & 0.36266333 & 2.23219538 & $\mathrm{H}$ & -1.17192888 & 0.99525338 & 1.84131861 \\
\hline $\mathrm{H}$ & -2.70574522 & 1.27248299 & 0.55987644 & $\mathrm{H}$ & -1.63556468 & 2.65114307 & -0.39671496 \\
\hline $\mathrm{C}$ & -3.49333143 & -0.61958128 & -1.24410331 & $\mathrm{C}$ & -4.98641396 & -0.60386312 & -0.28873011 \\
\hline $\mathrm{H}$ & -4.07361841 & 0.29662040 & -1.14134991 & $\mathrm{H}$ & -5.05909157 & -1.52279985 & -0.87219024 \\
\hline $\mathrm{H}$ & -4.11009121 & -1.44715261 & -0.88762057 & $\mathrm{H}$ & -5.60489941 & 0.15440910 & -0.76744050 \\
\hline $\mathrm{H}$ & -3.30735755 & -0.78483564 & -2.30618501 & $\mathrm{H}$ & -5.41315031 & -0.80853069 & 0.69580305 \\
\hline \multicolumn{8}{|c|}{$\mathrm{H}$} \\
\hline \multicolumn{4}{|c|}{$\mathrm{P} 2$} & \multicolumn{4}{|c|}{ R4 } \\
\hline \multicolumn{4}{|c|}{$\begin{array}{c}\text { Toluene } \\
\text { Same with P1 (Toluene) }\end{array}$} & \multicolumn{4}{|c|}{$\begin{array}{c}\mathrm{C}_{6} \mathrm{H}_{5} \mathrm{CHO} \\
\text { Same with } \mathrm{P} 4\left(\mathrm{C}_{6} \mathrm{H}_{5} \mathrm{CHO}\right) \text { in Table S1 }\end{array}$} \\
\hline \multirow{2}{*}{\multicolumn{4}{|c|}{$\begin{array}{c}\text { OH } \\
\text { Same with P2 }(\mathrm{OH})\end{array}$}} & \multirow{2}{*}{\multicolumn{4}{|c|}{$\begin{array}{c}\mathrm{CH}_{3} \\
\text { Same with R2( }\left(\mathrm{CH}_{3}\right) \text { in Table S1 }\end{array}$}} \\
\hline & & & & & & & \\
\hline \multicolumn{4}{|c|}{ TS9 } & \multicolumn{4}{|c|}{$\mathrm{P} 3$} \\
\hline $\mathrm{C}$ & -2.93295693 & 1.92344093 & 0.58581197 & \multicolumn{4}{|c|}{$\mathrm{C}_{6} \mathrm{H}_{5} \mathrm{CO}$} \\
\hline $\mathrm{C}$ & -1.99861777 & 0.89294875 & 0.49304259 & $\mathrm{C}$ & 0.17207827 & 0.10144792 & 0.63737428 \\
\hline $\mathrm{C}$ & -2.43063927 & -0.42090890 & 0.47535861 & $\mathrm{C}$ & 1.22714961 & -0.80496895 & 0.52256429 \\
\hline $\mathrm{C}$ & -3.79137516 & -0.70596862 & 0.54993910 & $\mathrm{C}$ & 0.95450741 & -2.15962791 & 0.44384268 \\
\hline $\mathrm{C}$ & -4.72177744 & 0.31973380 & 0.64224696 & $\mathrm{C}$ & -0.36246184 & -2.60904908 & 0.47699115 \\
\hline $\mathrm{C}$ & -4.29137754 & 1.63871884 & 0.66029382 & $\mathrm{C}$ & -1.41032279 & -1.70529282 & 0.58697402 \\
\hline $\mathrm{C}$ & -2.48088574 & 3.33622217 & 0.60560030 & $\mathrm{C}$ & -1.14254236 & -0.34731501 & 0.66735619 \\
\hline $\mathrm{H}$ & -0.94724363 & 1.14491749 & 0.43647397 & $\mathrm{C}$ & 0.43033928 & 1.56262410 & 0.70149404 \\
\hline $\mathrm{H}$ & -1.71292591 & -1.22764409 & 0.40358600 & $\mathrm{H}$ & 2.24500513 & -0.43897489 & 0.47996351 \\
\hline $\mathrm{H}$ & -4.12597847 & -1.73540509 & 0.53582782 & $\mathrm{H}$ & 1.76741624 & -2.86656618 & 0.34159338 \\
\hline $\mathrm{H}$ & -5.77776194 & 0.09153308 & 0.69983310 & $\mathrm{H}$ & -0.57149839 & -3.66885638 & 0.40221494 \\
\hline
\end{tabular}


Table S4. Cartesian coordinates $(\AA)$ of the reactants, highest energy TSs and products for the phenol formation pathways shown in Figure 8.

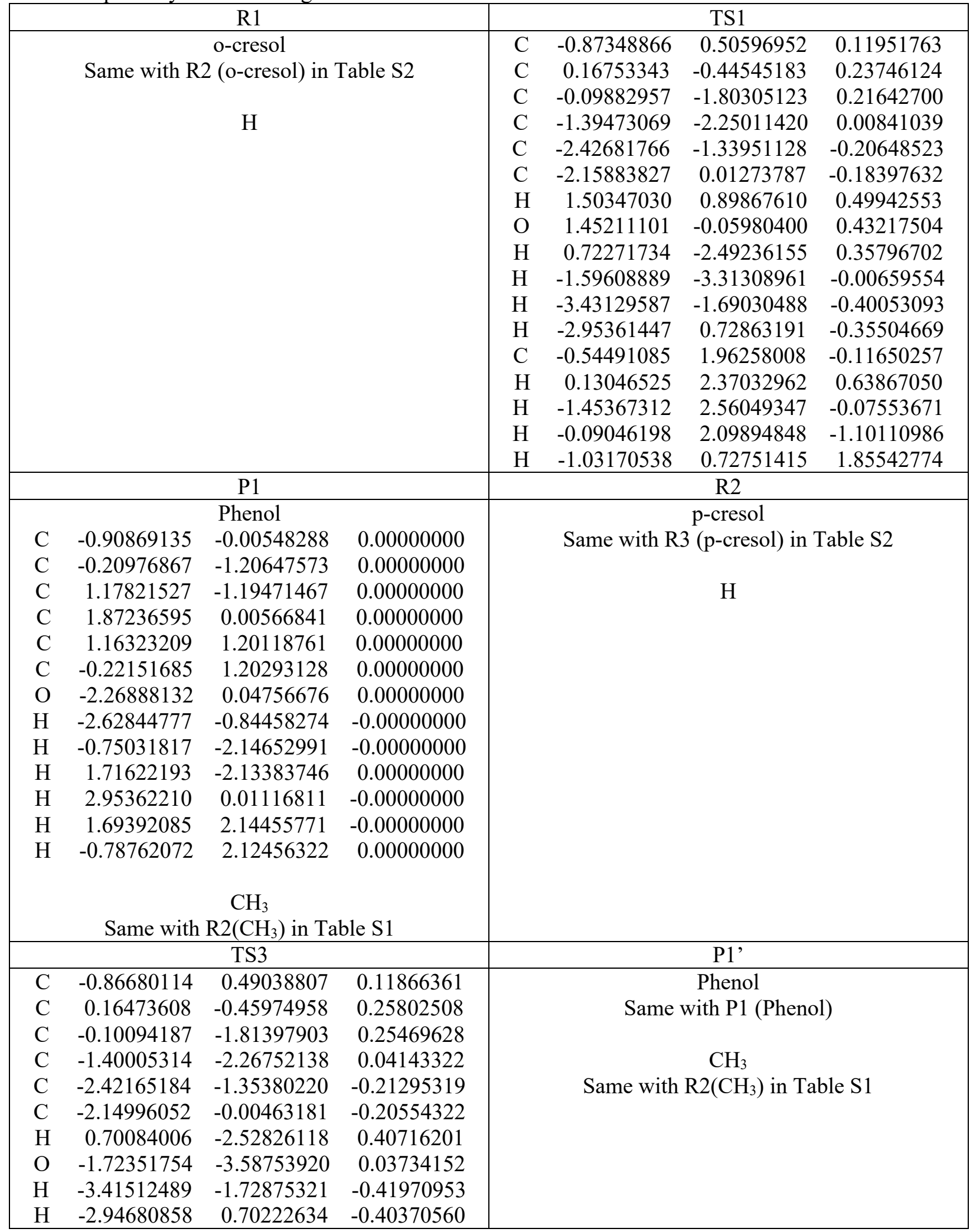




\begin{tabular}{|c|c|c|c|c|c|c|c|}
\hline $\mathrm{C}$ & -0.53281349 & 1.94438648 & -0.11883956 & & & & \\
\hline $\mathrm{H}$ & 0.33036324 & 2.24722099 & 0.47287139 & & & & \\
\hline $\mathrm{H}$ & -1.37095416 & 2.58556747 & 0.15178873 & & & & \\
\hline $\mathrm{H}$ & -0.30094621 & 2.11106229 & -1.17313015 & & & & \\
\hline $\mathrm{H}$ & -1.15945685 & 0.74711454 & 1.81869268 & & & & \\
\hline $\mathrm{H}$ & 1.17824519 & -0.11403515 & 0.42225638 & & & & \\
\hline $\mathrm{H}$ & -0.94259334 & -4.11464548 & 0.23184621 & & & & \\
\hline \multicolumn{4}{|c|}{ R3 } & \multicolumn{4}{|c|}{ TS5 } \\
\hline \multirow{3}{*}{\multicolumn{4}{|c|}{$\begin{array}{c}\text { Anisole } \\
\text { Same with R1(Anisole) }\end{array}$}} & $\mathrm{C}$ & 0.32000384 & 0.72736585 & 0.76735461 \\
\hline & & & & $\mathrm{C}$ & 1.00970364 & 1.02372336 & -0.40118867 \\
\hline & & & & $\mathrm{C}$ & 1.60699427 & 0.01674966 & -1.15074873 \\
\hline \multicolumn{4}{|c|}{ Phenoxy } & $\mathrm{C}$ & 1.51342547 & -1.29624856 & -0.72645450 \\
\hline $\mathrm{C}$ & -1.43829417 & 0.55322528 & 0.70823973 & $\mathrm{C}$ & 0.82471591 & -1.61112654 & 0.43953875 \\
\hline $\mathrm{C}$ & -0.31215191 & -0.33216491 & 0.49367386 & $\mathrm{C}$ & 0.23211545 & -0.59719485 & 1.17608535 \\
\hline $\mathrm{C}$ & -0.60604662 & -1.67684650 & 0.04250342 & $\mathrm{H}$ & -0.10581182 & 1.50663710 & 1.38198435 \\
\hline $\mathrm{C}$ & -1.89618945 & -2.07899404 & -0.16773912 & $\mathrm{O}$ & 1.18463433 & 2.29327488 & -0.88382816 \\
\hline $\mathrm{C}$ & -2.95809269 & -1.18791175 & 0.05265428 & $\mathrm{H}$ & 2.15204239 & 0.29025167 & -2.04418802 \\
\hline $\mathrm{C}$ & -2.71838856 & 0.12411807 & 0.48999557 & $\mathrm{H}$ & 1.98707509 & -2.07794809 & -1.30606353 \\
\hline $\mathrm{H}$ & -1.21467495 & 1.55690777 & 1.04536259 & $\mathrm{H}$ & 0.75499654 & -2.63810611 & 0.77169043 \\
\hline $\mathrm{O}$ & 0.85538322 & 0.04548549 & 0.68821889 & $\mathrm{H}$ & -0.29732615 & -0.83071512 & 2.09084463 \\
\hline $\mathrm{H}$ & 0.23788932 & -2.33523297 & -0.11667081 & $\mathrm{C}$ & 0.75689870 & 3.36353683 & -0.14415756 \\
\hline $\mathrm{H}$ & -2.10755038 & -3.08548737 & -0.50497967 & $\mathrm{H}$ & 0.91731590 & 4.27366352 & -0.71412367 \\
\hline $\mathrm{H}$ & -3.97488093 & -1.51676178 & -0.11690471 & $\mathrm{H}$ & 1.57532287 & 3.43990469 & 0.88844484 \\
\hline \multirow[t]{13}{*}{$\mathrm{H}$} & -3.55457282 & 0.79177397 & 0.65266573 & $\mathrm{H}$ & -0.24600855 & 3.26655436 & 0.27140194 \\
\hline & & & & $\mathrm{C}$ & 4.07408142 & 2.55043411 & 0.12431999 \\
\hline & & & & $\mathrm{C}$ & 3.29180670 & 2.32858658 & 1.26598608 \\
\hline & & & & $\mathrm{C}$ & 3.37489963 & 1.09090042 & 1.92352819 \\
\hline & & & & $\mathrm{C}$ & 4.22188330 & 0.10845821 & 1.45050156 \\
\hline & & & & $\mathrm{C}$ & 4.98108053 & 0.32926339 & 0.30310872 \\
\hline & & & & $\mathrm{C}$ & 4.90408897 & 1.55143189 & -0.35473675 \\
\hline & & & & $\mathrm{H}$ & 3.99592400 & 3.50674129 & -0.37746575 \\
\hline & & & & $\mathrm{O}$ & 2.46174908 & 3.26610970 & 1.72044325 \\
\hline & & & & $\mathrm{H}$ & 2.75427270 & 0.93299323 & 2.79584336 \\
\hline & & & & $\mathrm{H}$ & 4.28135777 & -0.84312046 & 1.96307218 \\
\hline & & & & $\mathrm{H}$ & 5.63094378 & -0.44938442 & -0.07438576 \\
\hline & & & & $\mathrm{H}$ & 5.49593782 & 1.72455943 & -1.24450243 \\
\hline \multicolumn{4}{|c|}{$\mathrm{P} 2$} & \multicolumn{4}{|c|}{$\mathrm{R} 4$} \\
\hline \multicolumn{4}{|c|}{$\begin{array}{c}\text { Anisyl } \\
\text { Same with P2 (Anisyl) in Table S1 }\end{array}$} & \multicolumn{4}{|c|}{$\begin{array}{c}\text { o-cresol } \\
\text { Same with R2 (o-cresol) in Table S2 }\end{array}$} \\
\hline \multicolumn{4}{|c|}{$\begin{array}{c}\text { Phenol } \\
\text { Same with P1 (Phenol) }\end{array}$} & \multicolumn{4}{|c|}{$\begin{array}{c}\text { Phenoxy } \\
\text { Same with R3 (Phenoxy) }\end{array}$} \\
\hline \multicolumn{4}{|c|}{ TS6 } & \multicolumn{4}{|c|}{ P3 } \\
\hline $\mathrm{C}$ & 0.52168047 & 0.60230029 & 1.05506504 & \multicolumn{4}{|c|}{$\mathrm{C}_{6} \mathrm{H}_{4} \mathrm{OCH}_{3}$} \\
\hline $\mathrm{C}$ & 0.99927050 & 1.67465961 & 0.23692672 & $\mathrm{C}$ & -1.51713312 & 0.59832567 & 0.78109324 \\
\hline $\mathrm{C}$ & 1.78548980 & 1.37761652 & -0.90716469 & $\mathrm{C}$ & -0.37462729 & -0.24981907 & 0.50870359 \\
\hline $\mathrm{C}$ & 2.14587665 & 0.07955351 & -1.19134998 & $\mathrm{C}$ & -0.62821704 & -1.58582258 & -0.02526838 \\
\hline $\mathrm{C}$ & 1.70702446 & -0.95591098 & -0.36917791 & $\mathrm{C}$ & -1.92591679 & -1.98084974 & -0.24564086 \\
\hline $\mathrm{C}$ & 0.88336217 & -0.68668121 & 0.72741908 & $\mathrm{C}$ & -3.00053358 & -1.12934673 & 0.03171349 \\
\hline $\mathrm{O}$ & 0.73533285 & 2.90170383 & 0.57036525 & $\mathrm{C}$ & -2.78848076 & 0.16192478 & 0.54605073 \\
\hline
\end{tabular}




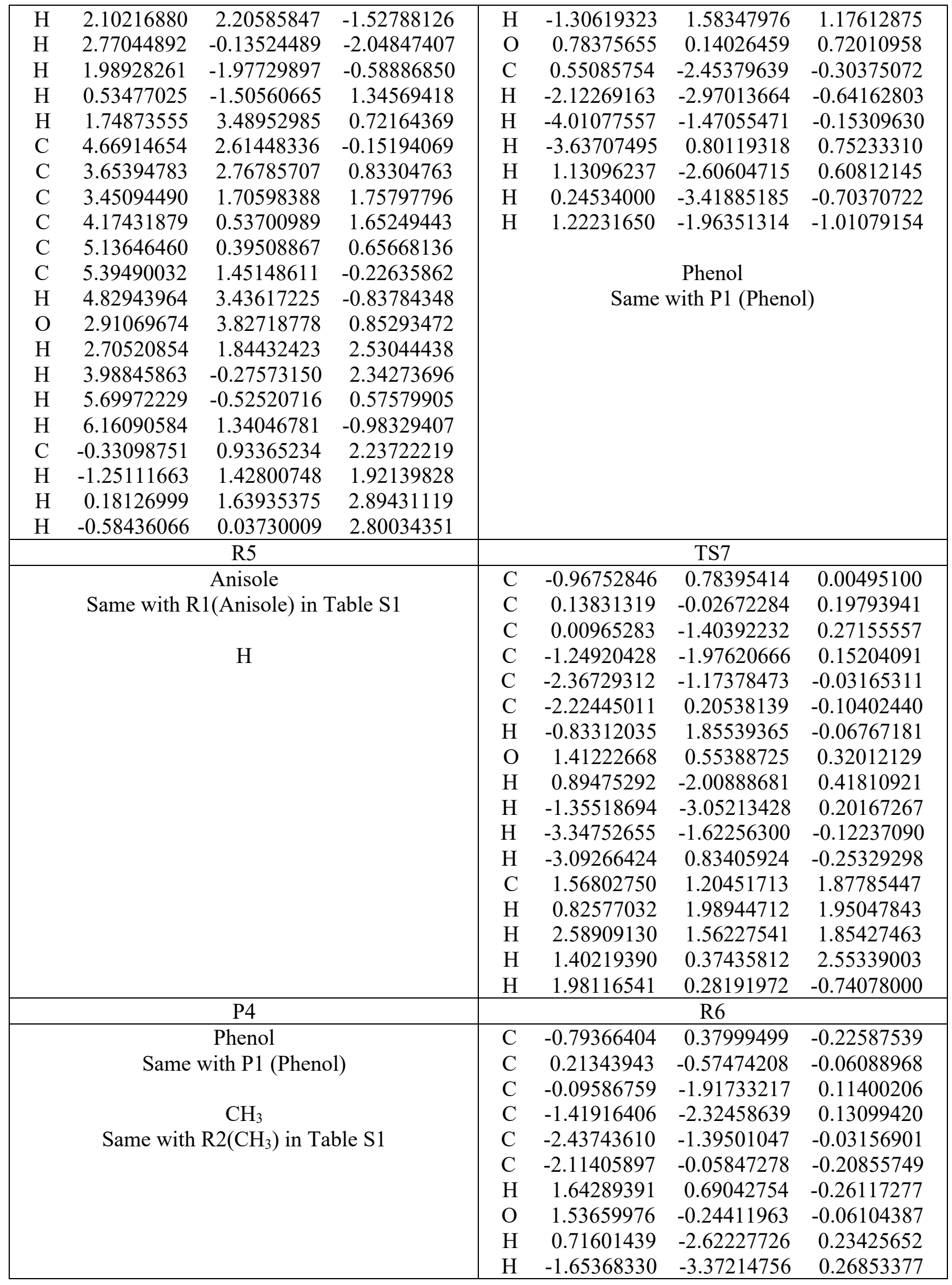




\begin{tabular}{|c|c|c|c|c|c|c|c|}
\hline & & & & $\begin{array}{l}\mathrm{H} \\
\mathrm{H} \\
\mathrm{C} \\
\mathrm{H} \\
\mathrm{H} \\
\mathrm{C} \\
\mathrm{H} \\
\mathrm{H} \\
\mathrm{H}\end{array}$ & $\begin{array}{r}-3.47263885 \\
-2.90333796 \\
-0.46050152 \\
-1.32298505 \\
0.34762409 \\
-0.08351602 \\
-0.91949540 \\
0.17295147 \\
0.76589429\end{array}$ & $\begin{array}{r}-1.70802605 \\
0.67344338 \\
1.84208727 \\
2.35820842 \\
1.98387766 \\
2.49035621 \\
2.42338443 \\
3.54198360 \\
1.98596835 \\
\end{array}$ & $\begin{array}{r}-0.02338991 \\
-0.33899435 \\
-0.36911574 \\
-0.79242045 \\
-1.09592903 \\
0.96640944 \\
1.66275012 \\
0.83599818 \\
1.43059659\end{array}$ \\
\hline \multicolumn{4}{|c|}{ TS8 } & \multicolumn{4}{|c|}{ P5 } \\
\hline $\mathrm{C}$ & -1.04918468 & 0.46813074 & 0.39013058 & \multicolumn{4}{|c|}{ Phenol } \\
\hline $\mathrm{C}$ & 0.04208175 & -0.45864734 & 0.63228571 & \multicolumn{4}{|c|}{ Same with P1 (Phenol) } \\
\hline $\mathrm{C}$ & 0.07924168 & -1.74783599 & 0.04919470 & & & & \\
\hline $\mathrm{C}$ & -1.12346625 & -2.23141408 & -0.38707352 & & & $\mathrm{C}_{2} \mathrm{H}_{4}$ & \\
\hline $\mathrm{C}$ & -2.32049298 & -1.45715594 & -0.36703160 & $\mathrm{C}$ & -0.78631568 & 0.05991683 & 0.00000000 \\
\hline $\mathrm{C}$ & -2.27979350 & -0.14076713 & -0.01718555 & $\mathrm{C}$ & 0.35867819 & -0.60119796 & 0.00000000 \\
\hline $\mathrm{H}$ & 0.42028004 & 2.85142899 & 1.23799622 & $\mathrm{H}$ & 1.31130576 & -0.08632394 & -0.00000000 \\
\hline $\mathrm{O}$ & 0.77994215 & -0.01187144 & 1.57879567 & $\mathrm{H}$ & -0.81679213 & 1.14234757 & -0.00000000 \\
\hline $\mathrm{H}$ & 0.97070575 & -2.35716534 & 0.10575373 & $\mathrm{H}$ & -1.73894620 & -0.45494696 & -0.00000000 \\
\hline $\mathrm{H}$ & -1.18113172 & -3.25785184 & -0.73033577 & $\mathrm{H}$ & 0.38915145 & -1.68363011 & 0.00000000 \\
\hline $\mathrm{H}$ & -3.24513960 & -1.91399407 & -0.69110394 & & & & \\
\hline $\mathrm{H}$ & -3.16056800 & 0.48258317 & -0.13132006 & & & & \\
\hline $\mathrm{C}$ & -0.75880045 & 1.83295357 & -0.27963617 & & & & \\
\hline $\mathrm{H}$ & -1.64525330 & 2.44798374 & -0.10945213 & & & & \\
\hline $\mathrm{H}$ & -0.69832999 & 1.67128205 & -1.35978281 & & & & \\
\hline $\mathrm{C}$ & 0.48046371 & 2.58520150 & 0.18207934 & & & & \\
\hline $\mathrm{H}$ & 0.58214265 & 3.50413489 & -0.39583284 & & & & \\
\hline $\mathrm{H}$ & 1.38559651 & 1.99499285 & 0.04569258 & & & & \\
\hline $\mathrm{H}$ & -0.35408536 & 0.72980726 & 1.58848011 & & & & \\
\hline
\end{tabular}


Table S5. Cartesian coordinates $(\AA)$ of the reactants, highest energy TSs and products for pathways of the formation of cresol and benzofuran shown in Figure 9.

\begin{tabular}{|c|c|c|c|c|c|c|c|}
\hline \multicolumn{4}{|c|}{ R1 } & \multicolumn{4}{|c|}{ TS5 } \\
\hline $\mathrm{C}$ & -0.86193639 & 0.59894866 & 0.34938210 & $\mathrm{C}$ & -0.82712084 & 0.47038358 & -0.12504378 \\
\hline $\mathrm{C}$ & 0.25297761 & -0.43861112 & 0.40436020 & $\mathrm{C}$ & 0.21114360 & -0.50406706 & -0.14817145 \\
\hline $\mathrm{C}$ & -0.08106437 & -1.82895696 & 0.06284975 & $\mathrm{C}$ & -0.03227176 & -1.88556802 & 0.17544630 \\
\hline $\mathrm{C}$ & -1.34609103 & -2.19014621 & -0.19038054 & $\mathrm{C}$ & -1.38414645 & -2.35193086 & 0.17062654 \\
\hline $\mathrm{C}$ & -2.45094156 & -1.23875630 & -0.15579869 & $\mathrm{C}$ & -2.38476944 & -1.44539320 & 0.00736801 \\
\hline $\mathrm{C}$ & -2.23074150 & 0.04865854 & 0.10876251 & $\mathrm{C}$ & -2.09199214 & -0.05037882 & -0.09878894 \\
\hline $\mathrm{H}$ & -0.84552509 & 1.10969114 & 1.31820667 & $\mathrm{O}$ & 1.38880420 & -0.36424315 & -0.62819737 \\
\hline $\mathrm{O}$ & 1.37927222 & -0.10669564 & 0.70214665 & $\mathrm{C}$ & -0.50156099 & 1.92007232 & -0.30428544 \\
\hline $\mathrm{H}$ & 0.73993051 & -2.53378487 & 0.04673542 & $\mathrm{H}$ & 0.18741120 & 2.26041842 & 0.47013041 \\
\hline $\mathrm{H}$ & -1.57236040 & -3.22367620 & -0.42663825 & $\mathrm{H}$ & -1.40392458 & 2.52804446 & -0.26112124 \\
\hline $\mathrm{H}$ & -3.45140314 & -1.60211825 & -0.35037574 & $\mathrm{H}$ & -0.00734655 & 2.08569860 & -1.26295578 \\
\hline $\mathrm{H}$ & -3.05331063 & 0.75571358 & 0.12613174 & $\mathrm{H}$ & -2.92736483 & 0.63626808 & -0.19385919 \\
\hline $\mathrm{C}$ & -0.50532764 & 1.64120996 & -0.72671950 & $\mathrm{H}$ & -3.41905117 & -1.76025033 & 0.00420430 \\
\hline $\mathrm{H}$ & 0.48904648 & 2.03782105 & -0.52947259 & $\mathrm{H}$ & 1.09945130 & -1.72910786 & -0.61272275 \\
\hline $\mathrm{H}$ & -1.22707546 & 2.45785761 & -0.72142309 & $\mathrm{H}$ & 0.64352381 & -2.29602623 & 0.92699492 \\
\hline $\mathrm{H}$ & -0.51353991 & 1.18080580 & -1.71566117 & $\mathrm{H}$ & -1.60386074 & -3.39563751 & 0.35629141 \\
\hline \multicolumn{4}{|c|}{$\mathrm{P} 1$} & \multicolumn{4}{|c|}{ TS6 } \\
\hline \multirow{16}{*}{\multicolumn{4}{|c|}{$\begin{array}{c}\text { o-cresol } \\
\text { Same with R2 (o-cresol) in Table S2 }\end{array}$}} & $\mathrm{C}$ & -0.77784693 & 0.53971171 & 0.12852328 \\
\hline & & & & $\mathrm{C}$ & 0.21335825 & -0.54088348 & 0.28863055 \\
\hline & & & & $\mathrm{C}$ & -0.01277918 & -1.86902869 & 0.00198737 \\
\hline & & & & $\mathrm{C}$ & -1.32475460 & -2.20220804 & -0.33142841 \\
\hline & & & & $\mathrm{C}$ & -2.36930633 & -1.24578094 & -0.11075887 \\
\hline & & & & $\mathrm{C}$ & -2.14813137 & 0.05588094 & 0.23760076 \\
\hline & & & & $\mathrm{H}$ & 0.06206466 & 0.92804140 & 1.19459963 \\
\hline & & & & $\mathrm{O}$ & 1.07685530 & 0.05099469 & 1.10182059 \\
\hline & & & & $\mathrm{H}$ & 0.75512040 & -2.61787152 & 0.14534096 \\
\hline & & & & $\mathrm{H}$ & -1.58525455 & -3.21162701 & -0.61366135 \\
\hline & & & & $\mathrm{H}$ & -3.39328909 & -1.58425021 & -0.22442105 \\
\hline & & & & $\mathrm{H}$ & -2.96710610 & 0.74189103 & 0.40771627 \\
\hline & & & & $\mathrm{C}$ & -0.47077000 & 1.63833284 & -0.86937290 \\
\hline & & & & $\mathrm{H}$ & 0.57187533 & 1.94843256 & -0.80572164 \\
\hline & & & & $\mathrm{H}$ & -1.11596382 & 2.50076485 & -0.70342147 \\
\hline & & & & $\mathrm{H}$ & -0.65952492 & 1.25556457 & -1.87736034 \\
\hline \multicolumn{4}{|c|}{$\mathrm{R} 2$} & \multicolumn{4}{|c|}{ TS9 } \\
\hline $\mathrm{C}$ & -0.84284425 & 0.56152880 & 1.14384973 & $\mathrm{C}$ & -3.17232633 & -0.10042956 & 1.44978476 \\
\hline $\mathrm{C}$ & 0.25449094 & -0.41565093 & 0.98515433 & $\mathrm{C}$ & -2.39740849 & -1.31337833 & 1.49267697 \\
\hline $\mathrm{C}$ & 0.01252738 & -1.52134192 & 0.03501241 & $\mathrm{C}$ & -2.38467240 & -2.19966245 & 0.38473520 \\
\hline $\mathrm{C}$ & -1.13155830 & -1.62457716 & -0.63678563 & $\mathrm{C}$ & -3.37634563 & -2.01879883 & -0.53590840 \\
\hline $\mathrm{C}$ & -2.25645018 & -0.64868534 & -0.51398319 & $\mathrm{C}$ & -4.33127356 & -0.94934642 & -0.49746776 \\
\hline $\mathrm{C}$ & -1.98188734 & 0.44599059 & 0.46544376 & $\mathrm{C}$ & -4.20250940 & 0.00894784 & 0.46311885 \\
\hline $\mathrm{H}$ & -0.66323072 & 1.37042725 & 1.84072471 & $\mathrm{H}$ & -2.60823894 & 0.80050856 & 1.69463813 \\
\hline $\mathrm{O}$ & 1.29512501 & -0.31555215 & 1.59986269 & $\mathrm{O}$ & -1.95711756 & -1.51389158 & 2.67507887 \\
\hline $\mathrm{H}$ & 0.81780022 & -2.23596835 & -0.07934073 & $\mathrm{H}$ & -1.71320093 & -3.04672360 & 0.35968888 \\
\hline $\mathrm{H}$ & -1.28255856 & -2.44481063 & -1.33229840 & $\mathrm{H}$ & -3.47155237 & -2.74035859 & -1.34157193 \\
\hline $\mathrm{H}$ & -3.13679719 & -1.20045364 & -0.15652990 & $\mathrm{H}$ & -2.91758251 & -0.50866151 & 2.75448728 \\
\hline
\end{tabular}




\begin{tabular}{|c|c|c|c|c|c|c|c|}
\hline $\mathrm{H}$ & -2.77119446 & 1.18008685 & 0.59736371 & $\mathrm{H}$ & -4.84211111 & 0.88410902 & 0.44592300 \\
\hline $\mathrm{C}$ & -2.62273812 & -0.06683307 & -1.89012051 & $\mathrm{C}$ & -5.39851284 & -0.89541793 & -1.55489218 \\
\hline $\mathrm{H}$ & -1.78072226 & 0.49419442 & -2.29612756 & $\mathrm{H}$ & -4.96342325 & -0.89019710 & -2.55620766 \\
\hline $\mathrm{H}$ & -3.47863984 & 0.60334140 & -1.80816936 & $\mathrm{H}$ & -6.00941992 & -0.00035828 & -1.44683218 \\
\hline $\mathrm{H}$ & -2.87686825 & -0.86426681 & -2.58860898 & $\mathrm{H}$ & -6.05872965 & -1.76303661 & -1.49134862 \\
\hline \multicolumn{4}{|c|}{ P2 } & \multicolumn{4}{|c|}{ R3 } \\
\hline \multirow{2}{*}{\multicolumn{4}{|c|}{ Same with R3 (p-cresol)) in Table S2 }} & \multicolumn{4}{|c|}{$\begin{array}{c}\text { Phenoxy } \\
\text { Same with R3 (Phenoxy) in Table S3 }\end{array}$} \\
\hline & & & & $\begin{array}{l}\mathrm{C} \\
\mathrm{C} \\
\mathrm{H} \\
\mathrm{H}\end{array}$ & $\begin{array}{l}-1.27305722 \\
-1.27305722 \\
-1.27305722 \\
-1.27305722\end{array}$ & $\begin{array}{r}\mathrm{C}_{2} \mathrm{H}_{2} \\
0.30356878 \\
-0.89094090 \\
1.36746824 \\
-1.95484030\end{array}$ & $\begin{array}{l}0.00000000 \\
0.00000000 \\
0.00000000 \\
0.00000000\end{array}$ \\
\hline \multicolumn{4}{|c|}{ TS10 } & \multicolumn{4}{|c|}{ P3 } \\
\hline $\mathrm{C}$ & -0.71169597 & 0.26573330 & 0.31177282 & \multicolumn{4}{|c|}{ Benzofuran } \\
\hline $\mathrm{C}$ & -0.08871916 & -0.88554126 & 0.82071668 & $\mathrm{C}$ & -0.90169805 & -1.41034830 & -0.00400062 \\
\hline $\mathrm{C}$ & -0.71048927 & -2.13362312 & 0.65307987 & $\mathrm{C}$ & 0.24016368 & -0.62773389 & -0.00426021 \\
\hline $\mathrm{C}$ & -1.93828118 & -2.21864200 & 0.02389984 & $\mathrm{C}$ & 0.22150241 & 0.76911455 & -0.00437851 \\
\hline $\mathrm{C}$ & -2.55592179 & -1.06924069 & -0.46214479 & $\mathrm{C}$ & -1.00990736 & 1.42771423 & -0.00418820 \\
\hline $\mathrm{C}$ & -1.93944585 & 0.17067155 & -0.31605753 & $\mathrm{C}$ & -2.16272163 & 0.66533530 & -0.00403442 \\
\hline $\mathrm{H}$ & -0.20826629 & 1.21584773 & 0.43636733 & $\mathrm{C}$ & -2.10988927 & -0.73539048 & -0.00394008 \\
\hline $\mathrm{O}$ & 1.05877745 & -0.79298031 & 1.46821034 & $\mathrm{H}$ & -0.84272557 & -2.48981214 & -0.00389369 \\
\hline $\mathrm{H}$ & -0.20614511 & -3.01087880 & 1.03753877 & $\mathrm{O}$ & 1.52093089 & -1.08133316 & -0.00455562 \\
\hline $\mathrm{H}$ & -2.42096996 & -3.18044806 & -0.09253915 & $\mathrm{H}$ & -1.06044149 & 2.50870991 & -0.00417688 \\
\hline $\mathrm{H}$ & -3.51670957 & -1.13976204 & -0.95515734 & $\mathrm{H}$ & -3.12727094 & 1.15549862 & -0.00399478 \\
\hline $\mathrm{H}$ & -2.42294216 & 1.06148303 & -0.69600600 & $\mathrm{H}$ & -3.03250527 & -1.30042458 & -0.00383733 \\
\hline $\mathrm{C}$ & 2.61982417 & -0.93732971 & 0.43702286 & $\mathrm{C}$ & 2.31661057 & 0.02367376 & -0.00490858 \\
\hline $\mathrm{C}$ & 2.47248411 & -1.11187148 & -0.76695824 & $\mathrm{C}$ & 1.60722983 & 1.16763830 & -0.00480642 \\
\hline $\mathrm{H}$ & 3.24012089 & -0.81156069 & 1.29733181 & $\mathrm{H}$ & 3.37535977 & -0.17140958 & -0.00524079 \\
\hline $\mathrm{H}$ & 2.11396360 & -1.25604892 & -1.75977421 & $\mathrm{H}$ & 2.00906253 & 2.16635728 & -0.00508387 \\
\hline & & & & \multicolumn{4}{|c|}{$\mathrm{H}$} \\
\hline
\end{tabular}


Table S6. Cartesian coordinates $(\AA)$ of the reactants, highest energy TSs and products for pathways of the formation of cyclopentadiene, styrene, bzenzyl, methylene-benzene and indene shown in Figure S6.

\begin{tabular}{|c|c|c|c|c|c|c|c|}
\hline \multicolumn{4}{|c|}{ R1 } & \multicolumn{4}{|c|}{ TS2 } \\
\hline \multirow{2}{*}{\multicolumn{4}{|c|}{ Same with R3 (Phenoxy) in Table S3 }} & $\mathrm{C}$ & 0.04311135 & 0.80044955 & -0.46905720 \\
\hline & & & & $\mathrm{C}$ & -0.17595747 & 0.00302252 & 0.71671849 \\
\hline & & & & & & & \\
\hline & & & & $\mathrm{C}$ & -2.50091171 & 0.04878559 & 0.19105768 \\
\hline & & & & $\mathrm{C}$ & -0.67612159 & -1.32063127 & 0.24921516 \\
\hline & & & & $\mathrm{H}$ & -1.48556495 & 1.90527344 & 0.84352428 \\
\hline & & & & $\mathrm{C}$ & -2.01509213 & -1.26674294 & 0.00214019 \\
\hline & & & & $\mathrm{H}$ & -3.51274729 & 0.38044789 & 0.01822301 \\
\hline & & & & $\mathrm{H}$ & -0.02479916 & -2.17100215 & 0.12151472 \\
\hline & & & & $\mathrm{H}$ & -2.60847139 & -2.09565711 & -0.35565862 \\
\hline & & & & $\mathrm{O}$ & 0.81670505 & 1.48141205 & -1.04489899 \\
\hline & & & & $\mathrm{H}$ & 0.47541294 & 0.08831178 & 1.58835340 \\
\hline \multicolumn{4}{|c|}{ P1 } & \multicolumn{4}{|c|}{ R2 } \\
\hline \multicolumn{4}{|c|}{$\mathrm{C}_{5} \mathrm{H}_{5}$} & $\mathrm{C}$ & -6.01070690 & 2.85359740 & 0.94085938 \\
\hline $\mathrm{C}$ & -4.87425041 & 1.16576815 & 0.92279208 & $\mathrm{C}$ & -7.17336035 & 3.75893331 & 0.62913871 \\
\hline $\mathrm{C}$ & -5.96090126 & 2.13447762 & 0.69196606 & $\mathrm{C}$ & -8.00255489 & 3.14743829 & -0.22411978 \\
\hline $\mathrm{C}$ & -6.98444033 & 1.49033129 & 0.06773134 & $\mathrm{C}$ & -6.32453346 & 1.62923110 & 0.12124263 \\
\hline $\mathrm{C}$ & -5.26521873 & -0.04232910 & 0.43279409 & $\mathrm{H}$ & -7.28593016 & 4.74290371 & 1.05958104 \\
\hline $\mathrm{H}$ & -5.92978525 & 3.17507529 & 0.97753733 & $\mathrm{C}$ & -7.47137403 & 1.81431556 & -0.54193443 \\
\hline $\mathrm{C}$ & -6.58042765 & 0.12729427 & -0.10865308 & $\mathrm{H}$ & -8.91949368 & 3.55424023 & -0.62618220 \\
\hline $\mathrm{H}$ & -7.92691565 & 1.91105437 & -0.24225838 & $\mathrm{H}$ & -5.69483948 & 0.75203860 & 0.10959042 \\
\hline $\mathrm{H}$ & -4.70385408 & -0.96218634 & 0.44232765 & $\mathrm{H}$ & -7.94485998 & 1.10821664 & -1.20906961 \\
\hline $\mathrm{H}$ & -7.17192411 & -0.64718944 & -0.57444888 & $\mathrm{H}$ & -5.07337713 & 3.30743909 & 0.60143447 \\
\hline \multirow[t]{6}{*}{$\mathrm{H}$} & -3.93464875 & 1.39624941 & 1.40179217 & $\mathrm{C}$ & -5.89224243 & 2.54426742 & 2.41630316 \\
\hline & & & & $\mathrm{C}$ & -4.82268190 & 2.75819826 & 3.12554789 \\
\hline & & $\mathrm{CO}$ & & $\mathrm{H}$ & -6.76862574 & 2.10967064 & 2.88825488 \\
\hline & \multirow{3}{*}{\multicolumn{3}{|c|}{ Same with P4 (CO) in Table S2 }} & $\mathrm{C}$ & -3.74759007 & 2.97352886 & 3.82577753 \\
\hline & & & & $\mathrm{H}$ & -3.59169173 & 3.91539383 & 4.33792448 \\
\hline & & & & $\mathrm{H}$ & -2.97991872 & 2.21481538 & 3.91983342 \\
\hline \multicolumn{4}{|c|}{ TS4 } & \multicolumn{4}{|c|}{$\mathrm{P} 2$} \\
\hline $\mathrm{C}$ & -6.09024239 & 3.15635228 & 0.96043497 & $\mathrm{C}$ & -4.98624468 & 2.46693444 & 0.58542305 \\
\hline $\mathrm{C}$ & -7.39183092 & 3.76883817 & 0.53177673 & $\mathrm{C}$ & -4.58195066 & 1.20316863 & 1.02226496 \\
\hline $\mathrm{C}$ & -8.05301571 & 2.91803360 & -0.26101854 & $\mathrm{C}$ & -6.13767052 & 2.55861330 & -0.19601864 \\
\hline $\mathrm{C}$ & -6.12150908 & 1.80628479 & 0.27576414 & $\mathrm{C}$ & -6.87437916 & 1.42918575 & -0.52240783 \\
\hline $\mathrm{H}$ & -7.72251511 & 4.74222851 & 0.86201710 & $\mathrm{C}$ & -5.31419039 & 0.07462755 & 0.69735837 \\
\hline $\mathrm{C}$ & -7.26023483 & 1.69568408 & -0.41859725 & $\mathrm{H}$ & -6.45917988 & 3.53205252 & -0.54759640 \\
\hline $\mathrm{H}$ & -9.02323818 & 3.08097839 & -0.70746285 & $\mathrm{C}$ & -6.46558857 & 0.18222658 & -0.07491564 \\
\hline $\mathrm{H}$ & -5.32887125 & 1.07944238 & 0.36541280 & $\mathrm{H}$ & -7.76667595 & 1.52400661 & -1.12761199 \\
\hline $\mathrm{H}$ & -7.55446577 & 0.84445983 & -1.01603305 & $\mathrm{H}$ & -4.98405933 & -0.89677364 & 1.04257369 \\
\hline $\mathrm{H}$ & -5.22680712 & 3.74013662 & 0.61651933 & $\mathrm{H}$ & -7.03484678 & -0.70238310 & -0.32873622 \\
\hline $\mathrm{C}$ & -6.04052401 & 2.78990221 & 2.41934586 & $\mathrm{C}$ & -4.24780035 & 3.69676590 & 0.91425371 \\
\hline $\mathrm{C}$ & -4.85984087 & 2.81733632 & 3.06200886 & $\mathrm{H}$ & -3.68001962 & 1.09979141 & 1.61152077 \\
\hline $\mathrm{H}$ & -5.37756729 & 3.87490320 & 3.20048404 & $\mathrm{C}$ & -3.25421047 & 3.81639528 & 1.78717160 \\
\hline $\mathrm{C}$ & -3.74899077 & 2.49658227 & 3.70753002 & $\mathrm{H}$ & -2.77742434 & 4.77221680 & 1.95607769 \\
\hline $\mathrm{H}$ & -3.20541024 & 3.22188592 & 4.29080486 & $\mathrm{H}$ & -2.88613749 & 2.97932792 & 2.36724043 \\
\hline $\mathrm{H}$ & -3.37764668 & 1.48378742 & 3.65507746 & $\mathrm{H}$ & -4.57952547 & 4.58330536 & 0.38174826 \\
\hline
\end{tabular}




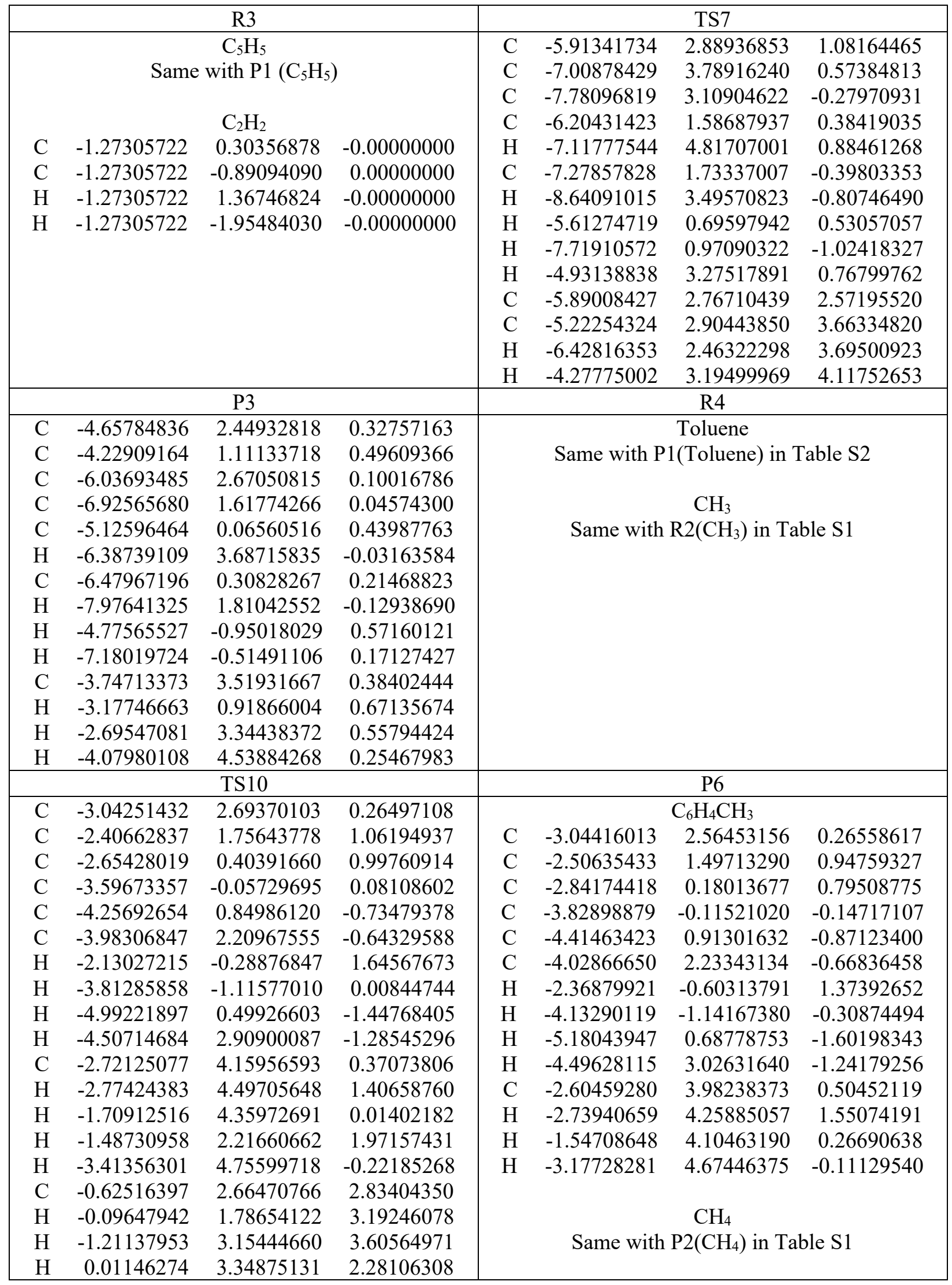




\begin{tabular}{|c|c|c|c|c|c|c|c|}
\hline \multicolumn{4}{|c|}{ R5 } & \multicolumn{4}{|c|}{ TS15 } \\
\hline \multicolumn{4}{|c|}{$\mathrm{C}_{5} \mathrm{H}_{5}$} & $\mathrm{C}$ & -7.16908264 & 1.61465013 & 2.31476259 \\
\hline \multirow{2}{*}{\multicolumn{4}{|c|}{ Same with $\mathrm{P} 1\left(\mathrm{C}_{5} \mathrm{H}_{5}\right)$ in Table S5 }} & $\mathrm{C}$ & -7.05815554 & 2.62636852 & 1.20301473 \\
\hline & & & & $\mathrm{C}$ & -6.92713308 & 1.90337765 & -0.12135683 \\
\hline \multicolumn{4}{|c|}{$\mathrm{C}_{4} \mathrm{H}_{6}$} & $\mathrm{C}$ & -7.48198366 & 0.34809527 & 1.55284822 \\
\hline $\mathrm{C}$ & -0.81533062 & 0.43539312 & 0.14152876 & $\mathrm{H}$ & -6.23177814 & 1.51253331 & 2.87422729 \\
\hline $\mathrm{C}$ & 0.29750192 & -0.22388032 & -0.16570094 & $\mathrm{C}$ & -7.37001896 & 0.50754905 & 0.23910831 \\
\hline $\mathrm{C}$ & 1.61544979 & 0.39596698 & -0.22776169 & $\mathrm{H}$ & -5.86258793 & 1.88161552 & -0.41326487 \\
\hline $\mathrm{C}$ & 2.72832441 & -0.26332325 & -0.53479606 & $\mathrm{H}$ & -7.74258518 & -0.57930249 & 2.04499507 \\
\hline $\mathrm{H}$ & -0.79374516 & 1.49546349 & 0.36659572 & $\mathrm{H}$ & -7.52766275 & -0.26788947 & -0.49931586 \\
\hline $\mathrm{H}$ & -1.77690399 & -0.05825147 & 0.17793208 & $\mathrm{H}$ & -7.94207144 & 1.88135564 & 3.03860211 \\
\hline $\mathrm{H}$ & 0.25289807 & -1.28620076 & -0.38783288 & $\mathrm{C}$ & -7.16683197 & 3.95435810 & 1.24097681 \\
\hline $\mathrm{H}$ & 1.66002035 & 1.45832157 & -0.00568855 & $\mathrm{C}$ & -7.18832493 & 4.71199369 & -0.00909440 \\
\hline $\mathrm{H}$ & 2.70667982 & -1.32339478 & -0.75986576 & $\mathrm{C}$ & -7.43501663 & 4.09485054 & -1.17610812 \\
\hline \multirow[t]{8}{*}{$\mathrm{H}$} & 3.68989301 & 0.23025033 & -0.57117170 & $\mathrm{C}$ & -7.67661047 & 2.64008808 & -1.20942175 \\
\hline & & & & $\mathrm{H}$ & -7.28057575 & 4.48298931 & 2.18019509 \\
\hline & & & & $\mathrm{H}$ & -9.83124352 & 2.19002748 & -0.50557941 \\
\hline & & & & $\mathrm{H}$ & -7.06569242 & 5.78705406 & 0.02634159 \\
\hline & & & & $\mathrm{H}$ & -7.53949070 & 4.66848373 & -2.08837199 \\
\hline & & & & $\mathrm{H}$ & -8.86667156 & 2.47183633 & -0.91908962 \\
\hline & & & & $\mathrm{H}$ & -7.58439302 & 2.18684363 & -2.19460964 \\
\hline & \multicolumn{3}{|c|}{ P5 } & \multicolumn{4}{|c|}{ P5-TS1 } \\
\hline \multicolumn{4}{|c|}{$\mathrm{C}_{6} \mathrm{H}_{4} \mathrm{C}_{3} \mathrm{H}_{5}$} & \multicolumn{4}{|c|}{ TS } \\
\hline $\mathrm{C}$ & -7.15758562 & 1.61116326 & 2.29368782 & $\mathrm{C}$ & -7.22399330 & 1.58851719 & 2.26571703 \\
\hline $\mathrm{C}$ & -7.00682640 & 2.62203217 & 1.18963778 & $\mathrm{C}$ & -7.20887661 & 2.60685992 & 1.16144466 \\
\hline $\mathrm{C}$ & -6.82507610 & 1.89099538 & -0.11376510 & $\mathrm{C}$ & -7.21221495 & 1.92620850 & -0.08664063 \\
\hline $\mathrm{C}$ & -7.57343578 & 0.37847653 & 1.51978898 & $\mathrm{C}$ & -7.42397118 & 0.29453933 & 1.52032089 \\
\hline $\mathrm{H}$ & -6.21351242 & 1.42661107 & 2.82274199 & $\mathrm{H}$ & -6.27343225 & 1.57760251 & 2.81320906 \\
\hline $\mathrm{C}$ & -7.41822481 & 0.53401369 & 0.21045139 & $\mathrm{C}$ & -7.41679287 & 0.48957705 & 0.20142823 \\
\hline $\mathrm{H}$ & -5.73908424 & 1.72227156 & -0.27461773 & $\mathrm{H}$ & -5.53983068 & 1.77120793 & -0.24777478 \\
\hline $\mathrm{H}$ & -7.92018795 & -0.52546304 & 2.00210071 & $\mathrm{H}$ & -7.52615118 & -0.66082418 & 2.01458406 \\
\hline $\mathrm{H}$ & -7.61661482 & -0.21675336 & -0.54305577 & $\mathrm{H}$ & -7.51087141 & -0.26949060 & -0.56201291 \\
\hline $\mathrm{H}$ & -7.88762140 & 1.92017782 & 3.04429960 & $\mathrm{H}$ & -8.00959778 & 1.77997422 & 3.00112367 \\
\hline $\mathrm{C}$ & -7.19824028 & 3.96139956 & 1.22971702 & $\mathrm{C}$ & -7.19432068 & 3.98108339 & 1.20441175 \\
\hline $\mathrm{C}$ & -7.34142303 & 4.69783878 & 0.02873512 & $\mathrm{C}$ & -7.26042223 & 4.69740391 & 0.00750570 \\
\hline $\mathrm{C}$ & -7.47191954 & 4.01282120 & -1.20532143 & $\mathrm{C}$ & -7.40458488 & 4.03522635 & -1.21290064 \\
\hline $\mathrm{C}$ & -7.30757952 & 2.66912389 & -1.29461169 & $\mathrm{C}$ & -7.42386246 & 2.65541482 & -1.27446783 \\
\hline $\mathrm{H}$ & -7.32649851 & 4.46549654 & 2.18122935 & $\mathrm{H}$ & -7.15811443 & 4.50638771 & 2.15133071 \\
\hline $\mathrm{H}$ & -7.47416115 & 5.76972294 & 0.06457776 & $\mathrm{H}$ & -7.24878550 & 5.77909851 & 0.02946653 \\
\hline $\mathrm{H}$ & -7.74088001 & 4.57868052 & -2.08889294 & $\mathrm{H}$ & -7.51598883 & 4.61381340 & -2.12090611 \\
\hline $\mathrm{H}$ & -7.43598080 & 2.15369248 & -2.23869348 & $\mathrm{H}$ & -7.53915071 & 2.14000940 & -2.21934009 \\
\hline \multicolumn{4}{|c|}{$\mathrm{H}_{2}$} & \multicolumn{4}{|c|}{$\begin{array}{c}\mathrm{H}_{2} \\
\left.\text { Same with P5( } \mathrm{H}_{2}\right) \text { in Table S1 }\end{array}$} \\
\hline \multicolumn{4}{|c|}{$\frac{\text { Same with } \mathrm{P} 5\left(\mathrm{H}_{2}\right) \text { in Table SI }}{\mathrm{P} 6}$} & & & & \\
\hline \multicolumn{4}{|c|}{ Indene } & & & & \\
\hline $\mathrm{C}$ & -7.22920084 & 1.58710873 & 2.26157427 & & & & \\
\hline $\mathrm{C}$ & -7.26140213 & 2.60524964 & 1.15450001 & & & & \\
\hline $\mathrm{C}$ & -7.38557386 & 1.93717933 & -0.07144418 & & & & \\
\hline $\mathrm{C}$ & -7.34738493 & 0.28530356 & 1.51513445 & & & & \\
\hline
\end{tabular}




\begin{tabular}{|crrr|r|}
\hline $\mathrm{H}$ & -6.30315208 & 1.63980854 & 2.84187460 & \\
$\mathrm{C}$ & -7.43506098 & 0.49752778 & 0.19758029 & \\
$\mathrm{H}$ & -7.35795832 & -0.67867333 & 2.00306082 & \\
$\mathrm{H}$ & -7.52845430 & -0.26241815 & -0.56563824 & \\
$\mathrm{H}$ & -8.05242920 & 1.72493470 & 2.96921206 & \\
$\mathrm{C}$ & -7.19072580 & 3.98441482 & 1.19907129 & \\
$\mathrm{C}$ & -7.24564505 & 4.69790220 & 0.00435832 & \\
$\mathrm{C}$ & -7.36991501 & 4.03511143 & -1.21239042 & \\
$\mathrm{C}$ & -7.44103861 & 2.64801335 & -1.26196420 & \\
$\mathrm{H}$ & -7.09471655 & 4.50676155 & 2.14347935 & \\
$\mathrm{H}$ & -7.19137526 & 5.77867651 & 0.02163042 & \\
$\mathrm{H}$ & -7.41162252 & 4.60774136 & -2.12997007 & \\
$\mathrm{H}$ & -7.53758526 & 2.13484931 & -2.21076298 & \\
& & & \\
& $\mathrm{H}$ & \\
& $\mathrm{H}$ & \\
\hline
\end{tabular}


Table S7. Cartesian coordinates $(\AA)$ of the reactants, highest energy TSs and products for pathways of the formation of methylcyclopentadienyl and methylcyclopentadiene shown in Figure S7.

\begin{tabular}{|c|c|c|c|c|c|c|c|}
\hline \multicolumn{4}{|c|}{$\mathrm{R} 1$} & \multicolumn{4}{|c|}{ TS1 } \\
\hline $\mathrm{C}$ & -1.46109688 & 0.54076755 & 0.70658529 & $\mathrm{C}$ & -4.62384796 & 2.20804620 & -0.50756931 \\
\hline $\mathrm{C}$ & -0.32707098 & -0.33708143 & 0.49955142 & $\mathrm{C}$ & -4.72982836 & 1.34745789 & 0.64984280 \\
\hline $\mathrm{C}$ & -0.62229776 & -1.67910326 & 0.04293210 & $\mathrm{C}$ & -6.01637030 & 2.20297909 & 0.57925808 \\
\hline $\mathrm{C}$ & -1.90962076 & -2.08149886 & -0.17454189 & $\mathrm{C}$ & -7.08842230 & 1.35403967 & 0.32561946 \\
\hline $\mathrm{C}$ & -2.99315405 & -1.20733321 & 0.03863380 & $\mathrm{C}$ & -5.24369669 & 0.03097636 & 0.18049178 \\
\hline $\mathrm{C}$ & -2.73486257 & 0.10776626 & 0.48013312 & $\mathrm{H}$ & -6.06657314 & 3.20880461 & 0.96881956 \\
\hline $\mathrm{H}$ & -1.24728096 & 1.54659259 & 1.04397392 & $\mathrm{C}$ & -6.60018110 & 0.04975406 & 0.04000802 \\
\hline $\mathrm{O}$ & 0.83753544 & 0.04256395 & 0.70097387 & $\mathrm{H}$ & -8.11996174 & 1.66425443 & 0.24736123 \\
\hline $\mathrm{H}$ & 0.22045180 & -2.33874559 & -0.11813577 & $\mathrm{H}$ & -4.59038162 & -0.80172485 & -0.03342662 \\
\hline $\mathrm{H}$ & -2.11339021 & -3.08893871 & -0.51824778 & $\mathrm{O}$ & -3.91465569 & 2.92971683 & -1.11555183 \\
\hline $\mathrm{H}$ & -3.57311177 & 0.77693462 & 0.63747633 & $\mathrm{H}$ & -4.01396322 & 1.41406739 & 1.47118282 \\
\hline $\mathrm{C}$ & -4.40117264 & -1.66803300 & -0.16813819 & $\mathrm{C}$ & -7.45038939 & -1.08727264 & -0.43067616 \\
\hline $\mathrm{H}$ & -4.44437170 & -2.54311943 & -0.81464523 & $\mathrm{H}$ & -6.87597990 & -2.01032066 & -0.48915523 \\
\hline $\mathrm{H}$ & -5.01435709 & -0.87818485 & -0.60275006 & $\mathrm{H}$ & -7.86239004 & -0.87839794 & -1.41913950 \\
\hline $\mathrm{H}$ & -4.85399008 & -1.93860817 & 0.79016834 & $\mathrm{H}$ & -8.28993988 & -1.24435449 & 0.24784747 \\
\hline \multicolumn{4}{|c|}{ P1 } & \multicolumn{4}{|c|}{ R2 } \\
\hline \multicolumn{4}{|c|}{ methylcyclopentadienyl } & $\mathrm{C}$ & -1.51713312 & 0.59832567 & 0.78109324 \\
\hline $\mathrm{C}$ & -1.19751859 & -0.69581509 & 0.93438071 & $\mathrm{C}$ & -0.37462729 & -0.24981907 & 0.50870359 \\
\hline $\mathrm{C}$ & -2.32352328 & 0.15189876 & 0.60105181 & $\mathrm{C}$ & -0.62821704 & -1.58582258 & -0.02526838 \\
\hline $\mathrm{C}$ & -3.13318515 & -0.54572111 & -0.23395704 & $\mathrm{C}$ & -1.92591679 & -1.98084974 & -0.24564086 \\
\hline $\mathrm{C}$ & -1.36669457 & -1.93682921 & 0.26117361 & $\mathrm{C}$ & -3.00053358 & -1.12934673 & 0.03171349 \\
\hline $\mathrm{H}$ & -2.46843791 & 1.15717030 & 0.96606290 & $\mathrm{C}$ & -2.78848076 & 0.16192478 & 0.54605073 \\
\hline $\mathrm{C}$ & -2.53310871 & -1.86687076 & -0.45207804 & $\mathrm{H}$ & -1.30619323 & 1.58347976 & 1.17612875 \\
\hline $\mathrm{H}$ & -4.05980062 & -0.20764980 & -0.67215741 & $\mathrm{O}$ & 0.78375655 & 0.14026459 & 0.72010958 \\
\hline $\mathrm{H}$ & -0.68509591 & -2.77109742 & 0.31670174 & $\mathrm{C}$ & 0.55085754 & -2.45379639 & -0.30375072 \\
\hline $\mathrm{H}$ & -2.95503449 & -2.64173174 & -1.07448936 & $\mathrm{H}$ & -2.12269163 & -2.97013664 & -0.64162803 \\
\hline $\mathrm{C}$ & -0.07465416 & -0.31544888 & 1.82562208 & $\mathrm{H}$ & -4.01077557 & -1.47055471 & -0.15309630 \\
\hline $\mathrm{H}$ & -0.44464725 & -0.06241751 & 2.82318687 & $\mathrm{H}$ & -3.63707495 & 0.80119318 & 0.75233310 \\
\hline $\mathrm{H}$ & 0.43228695 & 0.57595104 & 1.44600737 & $\mathrm{H}$ & 1.13096237 & -2.60604715 & 0.60812145 \\
\hline $\mathrm{H}$ & 0.65505129 & -1.11764801 & 1.91861367 & $\mathrm{H}$ & 0.24534000 & -3.41885185 & -0.70370722 \\
\hline & & & & $\mathrm{H}$ & 1.22231650 & -1.96351314 & -1.01079154 \\
\hline \multirow{2}{*}{\multicolumn{4}{|c|}{$\begin{array}{l}\mathrm{CO} \\
\mathrm{P} 4\left(\mathrm{H}_{2}\right) \text { in Table } \mathrm{S} 2\end{array}$}} & & & & \\
\hline & & & & & & & \\
\hline \multicolumn{4}{|c|}{ TS5 } & \multicolumn{4}{|c|}{$\mathrm{P} 1{ }^{\prime}$} \\
\hline $\mathrm{C}$ & -4.55748463 & 2.07584548 & -0.65181524 & \multirow{11}{*}{\multicolumn{4}{|c|}{ Same with P1 }} \\
\hline $\mathrm{C}$ & -4.74785995 & 1.42134428 & 0.62479550 & & & & \\
\hline $\mathrm{C}$ & -5.99559641 & 2.29863286 & 0.33337441 & & & & \\
\hline $\mathrm{C}$ & -7.06880569 & 1.42885005 & 0.11404087 & & & & \\
\hline $\mathrm{C}$ & -5.25909996 & 0.05770593 & 0.31415316 & & & & \\
\hline $\mathrm{C}$ & -6.59970379 & 0.09852046 & 0.06707661 & & & & \\
\hline $\mathrm{H}$ & -8.08061314 & 1.75190234 & -0.08132013 & & & & \\
\hline $\mathrm{H}$ & -4.61301517 & -0.80451912 & 0.26128694 & & & & \\
\hline $\mathrm{H}$ & -7.20199919 & -0.75799274 & -0.19929522 & & & & \\
\hline $\mathrm{O}$ & -3.79043603 & 2.67743683 & -1.31974709 & & & & \\
\hline $\mathrm{H}$ & -4.07347393 & 1.60773301 & 1.46293271 & & & & \\
\hline
\end{tabular}




\begin{tabular}{|c|c|c|c|c|c|c|c|}
\hline $\mathrm{C}$ & -6.09128332 & 3.74552608 & 0.71703070 & & & & \\
\hline $\mathrm{H}$ & -6.88951969 & 4.23316526 & 0.15828480 & & & & \\
\hline $\mathrm{H}$ & -5.16360712 & 4.28032923 & 0.51384795 & & & & \\
\hline $\mathrm{H}$ & -6.31214905 & 3.83881259 & 1.78234410 & & & & \\
\hline \multicolumn{4}{|c|}{ TS12 } & \multicolumn{4}{|c|}{$\mathrm{P} 2$} \\
\hline $\mathrm{C}$ & -7.41948318 & 3.05993032 & -0.19514467 & \multicolumn{4}{|c|}{ 1-methylcyclopentadiene } \\
\hline $\mathrm{C}$ & -6.90254641 & 0.95499432 & -1.33658695 & $\mathrm{C}$ & -0.95854145 & -0.38588840 & 0.74152207 \\
\hline $\mathrm{C}$ & -7.79076385 & 2.18984342 & -1.35655129 & $\mathrm{C}$ & -2.16544676 & 0.19573206 & 0.67361337 \\
\hline $\mathrm{C}$ & -8.97153568 & 1.83415127 & -0.53200889 & $\mathrm{C}$ & -3.07909203 & -0.65144217 & -0.10080937 \\
\hline $\mathrm{C}$ & -7.27559566 & 0.36051732 & 0.00663251 & $\mathrm{C}$ & -1.01375055 & -1.68723369 & -0.01031183 \\
\hline $\mathrm{C}$ & -8.50721073 & 0.81368601 & 0.35699967 & $\mathrm{H}$ & -2.43201685 & 1.14426494 & 1.12002623 \\
\hline $\mathrm{H}$ & -9.85688686 & 2.44154954 & -0.45070273 & $\mathrm{C}$ & -2.42779732 & -1.74879789 & -0.50570035 \\
\hline $\mathrm{H}$ & -6.73878670 & -0.44582513 & 0.48012790 & $\mathrm{H}$ & -4.11343431 & -0.41288081 & -0.30440167 \\
\hline $\mathrm{H}$ & -9.06329155 & 0.47322080 & 1.22001910 & $\mathrm{H}$ & -0.76670176 & -2.53608060 & 0.63687158 \\
\hline $\mathrm{O}$ & -7.42327595 & 3.84177899 & 0.65119296 & $\mathrm{H}$ & -2.82948852 & -2.56008339 & -1.09408009 \\
\hline $\mathrm{H}$ & -7.22491169 & 0.29945666 & -2.16052341 & $\mathrm{H}$ & -0.28943864 & -1.70511174 & -0.83224726 \\
\hline $\mathrm{C}$ & -7.91295528 & 2.98529577 & -2.63647223 & $\mathrm{C}$ & 0.26909229 & 0.10833995 & 1.42856598 \\
\hline $\mathrm{H}$ & -6.93964720 & 3.34338140 & -2.97183967 & $\mathrm{H}$ & 0.58161187 & -0.57820493 & 2.21943402 \\
\hline $\mathrm{H}$ & -8.58347321 & 3.83568144 & -2.51596093 & $\mathrm{H}$ & 0.09915920 & 1.08671200 & 1.87652028 \\
\hline $\mathrm{H}$ & -8.33211231 & 2.32192588 & -3.39259768 & $\mathrm{H}$ & 1.10583401 & 0.19272469 & 0.73096508 \\
\hline $\mathrm{H}$ & -5.85272598 & 1.20401061 & -1.47553861 & & & & \\
\hline & & & & \multicolumn{4}{|c|}{$\begin{array}{c}\mathrm{CO} \\
\text { ame with } \mathrm{P} 4\left(\mathrm{H}_{2}\right) \text { in Table S2 }\end{array}$} \\
\hline \multicolumn{4}{|c|}{ TS14 } & \multicolumn{4}{|c|}{$\mathrm{P} 3$} \\
\hline $\mathrm{C}$ & -3.24509931 & 0.35895011 & 0.10112648 & \multicolumn{4}{|c|}{ 2-methylcyclopentadiene } \\
\hline $\mathrm{C}$ & -2.18023944 & -0.62224096 & -1.15719330 & $\mathrm{C}$ & -0.91951871 & -0.32585865 & 0.63016284 \\
\hline $\mathrm{C}$ & -2.54817295 & -0.95471483 & 0.22754934 & $\mathrm{C}$ & -2.14221692 & 0.22168158 & 0.69090557 \\
\hline $\mathrm{C}$ & -3.62271023 & -2.02503395 & 0.27527201 & $\mathrm{C}$ & -3.09293866 & -0.69707423 & 0.04177481 \\
\hline $\mathrm{C}$ & -4.72378588 & -1.33198655 & -0.50218767 & $\mathrm{C}$ & -0.98701835 & -1.64987969 & -0.06752257 \\
\hline $\mathrm{C}$ & -4.51012373 & 0.00591393 & -0.48486191 & $\mathrm{C}$ & -2.44290876 & -1.77975631 & -0.40017480 \\
\hline $\mathrm{O}$ & -1.76854467 & -0.08433110 & -2.09887004 & $\mathrm{H}$ & -4.15343285 & -0.50361353 & -0.05149180 \\
\hline $\mathrm{H}$ & -1.65881503 & -1.05806601 & 0.84415227 & $\mathrm{H}$ & -0.64241725 & -2.47099113 & 0.57038891 \\
\hline $\mathrm{H}$ & -5.62232542 & -1.82573974 & -0.83613408 & $\mathrm{H}$ & -2.87197447 & -2.62579918 & -0.91641235 \\
\hline $\mathrm{C}$ & -2.61867142 & 1.70622909 & 0.17931509 & $\mathrm{H}$ & -0.36240852 & -1.67528594 & -0.96709043 \\
\hline $\mathrm{H}$ & -1.87494230 & 1.76735461 & 0.97547972 & $\mathrm{H}$ & -0.00926035 & 0.10727706 & 1.02019060 \\
\hline $\mathrm{H}$ & -3.37722921 & 2.46147823 & 0.38516963 & $\mathrm{C}$ & -2.53730631 & 1.52820599 & 1.29580534 \\
\hline $\mathrm{H}$ & -2.12448192 & 1.99085689 & -0.76441365 & $\mathrm{H}$ & -2.99516320 & 2.18001628 & 0.54849565 \\
\hline $\mathrm{H}$ & -3.91827488 & -2.18600321 & 1.32140875 & $\mathrm{H}$ & -1.67556834 & 2.04283953 & 1.71805453 \\
\hline $\mathrm{H}$ & -3.27572060 & -2.96788096 & -0.14011781 & $\mathrm{H}$ & -3.27364707 & 1.38238943 & 2.08906865 \\
\hline \multirow[t]{2}{*}{$\mathrm{H}$} & -5.19323349 & 0.74275017 & -0.88826019 & & & & \\
\hline & & & & \multicolumn{4}{|c|}{$\begin{array}{c}\mathrm{CO} \\
\text { Same with } \mathrm{P} 4\left(\mathrm{H}_{2}\right) \text { in Table S2 }\end{array}$} \\
\hline \multicolumn{4}{|c|}{ TS16 } & \multicolumn{4}{|c|}{ P4 } \\
\hline $\mathrm{C}$ & -7.20525408 & 2.97953081 & -0.09870271 & \multicolumn{4}{|c|}{ 5-methylcyclopentadiene } \\
\hline $\mathrm{C}$ & -6.95516253 & 0.83231336 & 1.16086507 & $\mathrm{C}$ & -0.82980740 & -0.46723041 & 0.44299740 \\
\hline $\mathrm{C}$ & -8.07219982 & 2.09841108 & 0.48472151 & $\mathrm{C}$ & -2.12409782 & 0.28388488 & 0.31210336 \\
\hline $\mathrm{C}$ & -8.69639683 & 1.22153223 & -0.57746565 & $\mathrm{C}$ & -3.09418941 & -0.55039656 & -0.08063765 \\
\hline $\mathrm{C}$ & -7.76624155 & -0.32523412 & 0.88944548 & $\mathrm{C}$ & -1.22721088 & -1.86423683 & 0.05981982 \\
\hline $\mathrm{H}$ & -8.74442482 & 2.56893730 & 1.21550798 & $\mathrm{H}$ & -2.22274518 & 1.33983183 & 0.52050823 \\
\hline $\mathrm{C}$ & -8.55543613 & -0.10723568 & -0.23572066 & $\mathrm{C}$ & -2.53140044 & -1.89842606 & -0.23883826 \\
\hline
\end{tabular}




\begin{tabular}{|c|c|c|c|c|c|c|c|}
\hline $\mathrm{H}$ & -9.36151028 & 1.62860155 & -1.31985581 & $\mathrm{H}$ & -4.12904406 & -0.29218668 & -0.25517640 \\
\hline $\mathrm{H}$ & -7.90705395 & -1.12760842 & 1.59833729 & $\mathrm{H}$ & -0.53761190 & -2.69640565 & 0.04701866 \\
\hline $\mathrm{H}$ & -9.11364079 & -0.88271493 & -0.74616009 & $\mathrm{H}$ & -3.09564972 & -2.76766372 & -0.54555732 \\
\hline $\mathrm{O}$ & -6.32175922 & 3.60766768 & -0.46672398 & $\mathrm{H}$ & -0.11204725 & -0.08118570 & -0.29313499 \\
\hline $\mathrm{C}$ & -6.46104097 & 1.12935019 & 2.55463314 & $\mathrm{C}$ & -0.20218472 & -0.36969891 & 1.83669782 \\
\hline $\mathrm{H}$ & -6.16402721 & 0.97361618 & 0.41471490 & $\mathrm{H}$ & -0.89800251 & -0.74751538 & 2.58655834 \\
\hline $\mathrm{H}$ & -5.70432329 & 0.39270425 & 2.83137250 & $\mathrm{H}$ & 0.04051988 & 0.66486180 & 2.08084869 \\
\hline $\mathrm{H}$ & -7.27207184 & 1.07648671 & 3.28014708 & $\mathrm{H}$ & 0.71488196 & -0.95729798 & 1.89029968 \\
\hline $\mathrm{H}$ & -5.99541616 & 2.11385393 & 2.61577702 & & & & \\
\hline & & & & \multicolumn{4}{|c|}{$\begin{array}{c}\mathrm{CO} \\
\text { Same with } \mathrm{P} 4\left(\mathrm{H}_{2}\right) \text { in Table S2 }\end{array}$} \\
\hline \multicolumn{4}{|c|}{ TS19 } & \multicolumn{4}{|c|}{ TS23 } \\
\hline $\mathrm{C}$ & -7.56425047 & 3.46666956 & -0.19483620 & $\mathrm{C}$ & -7.20034409 & 2.96130133 & -0.24205753 \\
\hline $\mathrm{C}$ & -6.66962528 & 1.16909742 & -0.55918527 & $\mathrm{C}$ & -7.01503754 & 0.88056791 & 1.18978858 \\
\hline $\mathrm{C}$ & -7.98315668 & 2.40725064 & -0.94844669 & $\mathrm{C}$ & -8.08429337 & 2.13930297 & 0.40079114 \\
\hline $\mathrm{C}$ & -9.06696510 & 1.66027272 & -0.20229581 & $\mathrm{C}$ & -8.65975094 & 1.22105908 & -0.65049815 \\
\hline $\mathrm{C}$ & -7.48105097 & -0.00791127 & -0.53367084 & $\mathrm{C}$ & -7.77314472 & -0.29115224 & 0.86948115 \\
\hline $\mathrm{C}$ & -8.76670361 & 0.31951833 & -0.09899451 & $\mathrm{C}$ & -8.50366592 & -0.10990150 & -0.30801320 \\
\hline $\mathrm{H}$ & -10.03883171 & 2.10780025 & -0.06526709 & $\mathrm{H}$ & -9.33632469 & 1.59413981 & -1.40272844 \\
\hline $\mathrm{H}$ & -7.22694349 & -0.92688078 & -1.03902948 & $\mathrm{H}$ & -8.00401497 & -1.06601739 & 1.58600700 \\
\hline $\mathrm{H}$ & -9.52181530 & -0.40805539 & 0.17338645 & $\mathrm{O}$ & -6.31054354 & 3.54621005 & -0.66222656 \\
\hline $\mathrm{O}$ & -7.04504728 & 4.25959969 & 0.44813603 & $\mathrm{H}$ & -6.79970217 & 1.10519433 & 2.23260045 \\
\hline $\mathrm{H}$ & -5.83650494 & 1.23772264 & -1.25680804 & $\mathrm{H}$ & -6.13633251 & 1.05364215 & 0.56487161 \\
\hline $\mathrm{C}$ & -8.26082611 & 2.65646935 & -2.42459321 & $\mathrm{H}$ & -8.78701019 & 2.67942786 & 1.04783940 \\
\hline $\mathrm{H}$ & -7.49073172 & 3.26338744 & -2.90046334 & $\mathrm{C}$ & -9.19166756 & -1.21676254 & -1.04617822 \\
\hline $\mathrm{H}$ & -9.23472214 & 3.12685442 & -2.55554271 & $\mathrm{H}$ & -8.48673916 & -1.73031223 & -1.70175290 \\
\hline $\mathrm{H}$ & -8.28554153 & 1.67204821 & -2.89362073 & $\mathrm{H}$ & -9.57852077 & -1.95485830 & -0.34227601 \\
\hline $\mathrm{H}$ & -6.40515184 & 1.56681061 & 0.42363811 & $\mathrm{H}$ & -10.01588821 & -0.84459990 & -1.65368319 \\
\hline \multicolumn{4}{|c|}{ P3' } & \multicolumn{4}{|c|}{ TS26 } \\
\hline \multirow{16}{*}{\multicolumn{4}{|c|}{ Same with P3 }} & $\bar{C}$ & -3.27852917 & 0.38216808 & 0.23684993 \\
\hline & & & & $\mathrm{C}$ & -2.25073671 & -0.64115393 & -1.13629401 \\
\hline & & & & $\mathrm{C}$ & -2.58346581 & -0.92766833 & 0.28188583 \\
\hline & & & & $\mathrm{C}$ & -3.67102766 & -1.98395038 & 0.34037614 \\
\hline & & & & $\mathrm{C}$ & -4.77454138 & -1.26724982 & -0.41903111 \\
\hline & & & & $\mathrm{C}$ & -4.54679203 & 0.07295118 & -0.34880576 \\
\hline & & & & $\mathrm{O}$ & -1.85441315 & -0.16118172 & -2.10839987 \\
\hline & & & & $\mathrm{H}$ & -1.67150807 & -1.06028450 & 0.85707432 \\
\hline & & & & $\mathrm{H}$ & -2.79892826 & 1.33873427 & 0.33834067 \\
\hline & & & & $\mathrm{H}$ & -5.23906231 & 0.82076538 & -0.71393639 \\
\hline & & & & $\mathrm{H}$ & -3.96525621 & -2.14738631 & 1.38786137 \\
\hline & & & & $\mathrm{H}$ & -3.35011959 & -2.93216419 & -0.08766641 \\
\hline & & & & $\mathrm{C}$ & -6.02336979 & -1.95875645 & -0.84475356 \\
\hline & & & & $\mathrm{H}$ & -6.72455215 & -1.25671172 & -1.29551196 \\
\hline & & & & $\mathrm{H}$ & -6.51997471 & -2.42900968 & 0.01201126 \\
\hline & & & & $\mathrm{H}$ & -5.82633352 & -2.75218201 & -1.56959081 \\
\hline \multicolumn{8}{|c|}{ P2' } \\
\hline \multicolumn{4}{|c|}{ Same with P2 } & & & & \\
\hline
\end{tabular}




\section{References}

(1) NIST Mass Spectrometry Data Center, W. E. W., director "Mass Spectra" in NIST Chemistry WebBook, NIST Standard Reference Database Number 69, Eds. P.J. Linstrom and W.G. Mallard, National Institute of Standards and Technology, Gaithersburg MD, 20899, https://doi.org/10.18434/T4D303, (retrieved August 30, 2020). 